\title{
SPECIAL POLYNOMIALS RELATED TO THE SUPERSYMMETRIC EIGHT-VERTEX MODEL: A SUMMARY
}

\author{
HJALMAR ROSENGREN
}

\begin{abstract}
We introduce and study symmetric polynomials, which as very special cases include polynomials related to the supersymmetric eight-vertex model, and other elliptic lattice models with $\Delta= \pm 1 / 2$. There is also a close relation to affine Lie algebra characters. After a natural change of variables, our polynomials satisfy a non-stationary Schrödinger equation with elliptic potential, which is related to the Knizhnik-Zamolodchikov-Bernard equation and to the canonical quantization of Painlevé VI. Moreover, specializations of our polynomials can be identified with tau functions of Painlevé VI, obtained from one of Picard's algebraic solutions by acting with a four-dimensional lattice of Bäcklund transformations. In the present work, our results on these topics are summarized with a minimum of technical details.
\end{abstract}

\section{INTRODUCTION}

In the present work, we introduce and study certain symmetric polynomials. Numerous special cases have appeared in connection with elliptic solvable lattice models, at the special parameter values usually denoted $\Delta= \pm 1 / 2$. We show that, up to a change of variables, our polynomials satisfy a Schrödinger equation with elliptic potential and that specializations of the polynomials are tau functions of Painlevé VI. For very special cases, these properties have been conjectured by Bazhanov and Mangazeev [BM1, BM2, MB]. The rigorous proofs of our main results contain many technical steps. For this reason, we have chosen to write the present summary, where the main results and ideas are described with a minimum of details. Complete proofs can be found in our series of preprints [R3, R4, R5].

The values $\Delta= \pm 1 / 2$ are truly exceptional. An intriguing feature is that exact results can be obtained not only in the infinite lattice limit but already on finite lattices. One explanation why $\Delta=-1 / 2$ is special comes from the limit to the massive sine-Gordon model, when it becomes a condition for supersymmetry [FS]. Recently, Hagendorf and Fendley $[\mathrm{HF}]$ implemented this supersymmetry on the finite lattice. Thus, we refer to $\Delta=-1 / 2$ as the supersymmetric case.

The six-vertex model with $\Delta=1 / 2$ contains the combinatorial ice model, where all states have equal weight. This was used by Kuperberg $[\mathrm{Ku}]$ in his simple proof of the alternating sign matrix theorem, which enumerates the states with domain wall boundary conditions. The combinatorics of the XXZ and six-vertex models at $\Delta=-1 / 2$ is also very rich, see [Z1] for a survey. It seems quite interesting to extend results in this area to the elliptic regime, but so far only the first steps have been taken. 
In [BM1, Bazhanov and Mangazeev found that the ground state eigenvalue of Baxter's $Q$-operator for the supersymmetric periodic XYZ chain of odd length can be expressed in terms of certain polynomials, which appear to have positive integer coefficients and thus call for a combinatorial interpretation. The papers [MB and [RS] deal with ground state eigenvectors of the Hamiltonian for the same chain. Certain components of these eigenvectors, as well as certain sums of components, again seem to be described by polynomials with positive coefficients. The same polynomials appear for other supersymmetric spin chains $[\mathrm{BH}, \overline{\mathrm{FH}}, \mathrm{H}]$. A rigorous investigation of the supersymmetric XYZ chain was recently initiated by Zinn-Justin [Z2].

As was noted in [MB], there are striking parallels between the work outlined above and our previous investigation of the 8VSOS and three-colour models R1, R2]. Just as the six-vertex model contains the combinatorial ice model when $\Delta=1 / 2$, the corresponding combinatorial specialization of the 8 VSOS model is the three-colour model. Extending Kuperberg's work to the elliptic regime, we expressed the domain wall partition function for the three-colour model in terms of certain special polynomials, which again conjecturally have positive integer coefficients.

In the the present work, we explain the relations between various polynomials introduced in [BM1, MB, R2, Z2, by identifying them as special cases of a more general family of functions. We stress that, although the underlying physical models are closely related [B1, B2], it is not clear why objects as different as domain wall partition functions, eigenvalues of the $Q$-operator and eigenvectors of the Hamiltonian should lead to related special functions.

To be more precise, we define for each non-negative integer $m$ a four-dimensional lattice $T_{n}^{(\mathbf{k})}$ of symmetric rational functions in $m$ variables, depending also on a parameter $\zeta$. The indices $n \in \mathbb{Z}$ and $\mathbf{k}=\left(k_{0}, k_{1}, k_{2}, k_{3}\right) \in \mathbb{Z}^{4}$ satisfy $|\mathbf{k}|+m=2 n$. Since the denominator is elementary, $T_{n}^{(\mathbf{k})}$ are essentially symmetric polynomials.

After a natural change of variables, $T_{n}^{(\mathbf{k})}$ is a multivariable theta function, which is closely related to affine Lie algebra characters. In fact, the theta function corresponding to $T_{n}^{(0,0,0,0)}$ can be identified with a character of the affine Lie algebra of type $C_{2 n}^{(1)}$. If all the indices $k_{i}$ are non-negative, $T_{n}^{(\mathbf{k})}$ is obtained from $T_{n}^{(0,0,0,0)}$ by specializing some of the variables to half-periods. In the general case, it can be obtained from the character $T_{n+\sum_{i} \max \left(-k_{i}, 0\right)}^{(0,0,0,0)}$ through a slightly more complicated procedure. This link to affine Lie algebras is not used to obtain our results, but may provide some explanation for the ubiquity of the functions $T_{n}^{(\mathbf{k})}$ in the context of solvable models.

Our first main result is that $T_{n}^{(\mathbf{k})}$ satisfies a certain algebraic differential equation, see Theorem 3.2. Special cases have been obtained by Bazhanov and Mangazeev [BM1, MB] (without complete proof) and Zinn-Justin [Z2]. Up to a change of 
variables, this equation is a non-stationary Schrödinger equation with elliptic potential, see Theorem 3.1. When $m=1$, it takes the form

$$
\psi_{t}=\frac{1}{2} \psi_{x x}-V \psi
$$

where $V$ is the Darboux potential [D, $\mathrm{D}]$

$$
V(x, t)=\sum_{j=0}^{3} \frac{k_{j}\left(k_{j}+1\right)}{2} \wp\left(x-\gamma_{j} \mid 1,2 \pi \mathrm{i} t\right),
$$

with $\gamma_{j}$ the four half-periods of the $\wp$-function. The $m$-variable case is simply the equation for $m$ non-interacting particles with the same potential.

The equation (1.1) has appeared in the literature in several contexts. It is a canonical quantization of Painlevé VI, and has been studied from this viewpoint by Nagoya [N1, [N2], Suleimanov [S1, [S2] and Zabrodin and Zotov [ZZ], see also [CD, No, ZS]. Under some extra condition on the parameters, it is the one-dimensional case of the Knizhnik-Zamolodchikov-Bernard heat equation satisfied by conformal blocks of Wess-Zumino-Witten theory on a torus [Be, EK]. The general case also appears in conformal field theory [FLNO. More precisely, (1.1) corresponds to a theory with central charge $c=1$, a case known to have close connections to Painlevé VI, see e.g. [ER, GIL]. Recently, Kolb [Ko] identified the corresponding Schrödinger operator with the radial part of the Casimir operator for the affine Lie algebra $\widehat{s l}_{2}$. Finally, we mention the paper [LT], where a more general equation, representing interacting particles, is used to study the Inozemtsev model.

An important application of the Schrödinger equation is that it implies bilinear relations for $T_{n}^{(\mathbf{k})}$, see Theorem 3.3. This is used to obtain our next main result, Theorem 4.2, where the functions $T_{n}^{(\mathbf{k})}$ with $m=0$ are identified with tau functions of Painlevé VI, obtained from one of Picard's solutions by acting with a four-dimensional lattice of Bäcklund transformations. For particular lines in the lattice, this has been conjectured by Bazhanov and Mangazeev [BM2]. As an application, we obtain a new quadratic differential equation for $T_{n}^{(\mathbf{k})}$ when $m=0$, see Proposition 4.5. Note that our tau functions can be obtained from $m=1$ instances of $T_{n}^{(\mathbf{k})}$, that is, from solutions to (1.1), by specializing the variable to a half period. A similar observation was made in [N2] for another class of solutions. Presumably, this phenomenon is linked to the relation between (1.1) and the Lax representation of Painlevé VI described in [CD, S1, ZZ].

In the final \$5, we explain the relation between $T_{n}^{(\mathbf{k})}$ and various polynomials introduced in [BM1, MB, R2, Z2] and also occurring in [BM2, [BH, $[\mathrm{FH},[\mathrm{H}, \mathrm{RS}]$. To be precise, for the polynomials related to eigenvalues of the $Q$-operator and to domain wall partition functions, these relations have been rigorously proved. However, for polynomials related to eigenvectors of the Hamiltonian, the identification with our polynomials $T_{n}^{(\mathbf{k})}$ is still based on empirical observation. A partially rigorous result exists only for the "sum rule" giving the square norm of 
the eigenvector, which was recently proved by Zinn-Justin [Z2], assuming a certain conjecture.

\section{Definition And FUndamental PROPERTIES of $T_{n}^{(\mathbf{k})}$}

2.1. Notation. Throughout the paper,

$$
\omega=e^{2 \pi \mathrm{i} / 3}
$$

We fix $\tau$ in the upper half-plane, and write $p=e^{\pi \mathrm{i} \tau}$. The four half-periods in $\mathbb{C} /(\mathbb{Z}+\tau \mathbb{Z})$ will be denoted

$$
\gamma_{0}=0, \quad \gamma_{1}=\frac{\tau}{2}, \quad \gamma_{2}=\frac{\tau}{2}+\frac{1}{2}, \quad \gamma_{3}=\frac{1}{2}
$$

We will use the notation

$$
\theta(x ; p)=\prod_{j=0}^{\infty}\left(1-p^{j} x\right)\left(1-\frac{p^{j+1}}{x}\right) .
$$

Repeated variables are used as a short-hand for products; for instance,

$$
\theta\left(a, b^{ \pm} ; p\right)=\theta(a ; p) \theta(b ; p) \theta\left(b^{-1} ; p\right) .
$$

Finally, the Vandermonde product is denoted

$$
\Delta(x)=\Delta\left(x_{1}, \ldots, x_{n}\right)=\prod_{1 \leq i<j \leq n}\left(x_{j}-x_{i}\right) .
$$

2.2. Anti-symmetric theta functions. For $n$ a non-negative integer, we denote by $\Theta_{n}$ the space of entire functions $f$ such that

$$
\begin{gathered}
f(z+1)=f(z), \quad f(z+\tau)=e^{-6 \pi \mathrm{i} n(\tau+2 z)} f(z), \quad f(-z)=-f(z), \\
f(z)+f\left(z+\frac{1}{3}\right)+f\left(z-\frac{1}{3}\right)=0 .
\end{gathered}
$$

This space has dimension $2 n$, an explicit basis being

$$
f_{j}(z)=e^{2 \pi \mathrm{i}(j-3 n) z} \theta\left(-p^{2 j} e^{12 \pi \mathrm{i} n z} ; p^{12 n}\right)-e^{2 \pi \mathrm{i}(3 n-j) z} \theta\left(-p^{2 j} e^{-12 \pi \mathrm{i} n z} ; p^{12 n}\right),
$$

where $1 \leq j \leq 3 n-1$ and $3 \nmid j$.

We are interested in the one-dimensional space $\Theta_{n}^{\wedge 2 n}$, which we realize as a space of anti-symmetric functions. One way to construct an element of this space is as the alternant

$$
\operatorname{det}_{1 \leq i \leq 2 n, 1 \leq j \leq 3 n-1,3 \nmid j}\left(f_{j}\left(z_{i}\right)\right)
$$


As we explain in \$2.8, (2.3) is essentially a character for the affine Lie algebra of type $C_{2 n}^{(1)}$. However, for our purposes a more useful generator of the same space is

$$
\begin{aligned}
\prod_{j=1}^{2 n} e^{-2 \pi \mathrm{i} z_{j}} \theta\left(e^{4 \pi \mathrm{i} z_{j}} ; p^{2}\right) \prod_{i, j=1}^{n} e^{-6 \pi \mathrm{i} z_{n+j}} & \theta\left(e^{6 \pi \mathrm{i}\left(z_{n+j} \pm z_{i}\right)} ; p^{6}\right) \\
& \times \operatorname{det}_{1 \leq i, j \leq n}\left(\frac{e^{-2 \pi \mathrm{i} z_{n+j}} \theta\left(e^{2 \pi \mathrm{i}\left(z_{n+j} \pm z_{i}\right)} ; p^{2}\right)}{e^{-6 \pi \mathrm{i} z_{n+j}} \theta\left(e^{6 \pi \mathrm{i}\left(z_{n+j} \pm z_{i}\right)} ; p^{6}\right)}\right) .
\end{aligned}
$$

The function (2.4) is analogous to the domain wall partition function for the six-vertex model [ICK, O, St], which for $\Delta=1 / 2$ can be expressed as the Schur polynomial

$$
Z=s_{(n-1, n-1, \ldots, 1,1,0,0)}\left(z_{1}, \ldots, z_{2 n}\right) .
$$

The usual determinant formula for this polynomial can be written

$$
Z=\frac{\operatorname{det}_{1 \leq i \leq 2 n, 1 \leq j \leq 3 n-1,3 \nmid j}\left(z_{i}^{j-1}\right)}{\Delta(z)},
$$

whereas the Izergin-Korepin formula gives

$$
Z=\frac{\prod_{i, j=1}^{n}\left(z_{n+j}^{3}-z_{i}^{3}\right)}{\Delta(z)} \operatorname{det}_{1 \leq i, j \leq n}\left(\frac{z_{n+j}-z_{i}}{z_{n+j}^{3}-z_{i}^{3}}\right) .
$$

After multiplication by $\Delta(z)$, these expressions are clearly analogous to (2.3) and (2.4). Even more to the point, the case $p=0$ of (2.4) is essentially the Tsuchiya determinant [T] (with $\Delta=1 / 2$ ), which is the partition function for the six-vertex model on a rectangle bounded by one reflecting edge and three domain walls. Recently, Filali generalized this to the elliptic level, interpreting (2.4) with general $p$ as a partition function for the 8 VSOS model $[\mathrm{F}]$. The genuine domain wall partition function for the 8VSOS model is more complicated [R1].

2.3. Uniformization. With a slight modification of the notation used in [R2], we will write

$$
\begin{aligned}
x(z) & =\frac{\theta\left(-p \omega ; p^{2}\right)^{2} \theta\left(\omega e^{ \pm 2 \pi \mathrm{i} z} ; p^{2}\right)}{\theta\left(-\omega ; p^{2}\right)^{2} \theta\left(p \omega e^{ \pm 2 \pi \mathrm{i} z} ; p^{2}\right)} \\
\zeta(\tau) & =\frac{\omega^{2} \theta\left(-1,-p \omega ; p^{2}\right)}{\theta\left(-p,-\omega ; p^{2}\right)}
\end{aligned}
$$

The function $x$ generates the field of even elliptic function with periods 1 and $\tau$. Moreover, as we discuss in 2.5 , $\zeta$ generates the field of modular functions for the group $\Gamma_{0}(6,2) \simeq \Gamma_{0}(12)$. Thus, if a function of $(z, \tau)$ has the appropriate elliptic and modular behaviour, it is automatically a rational function of $(x, \zeta)$. We refer to the change of variables from $(z, \tau)$ to $(x, \zeta)$ as uniformization. 
Uniformizing the determinant (2.4), we are led to define

$$
T\left(x_{1}, \ldots, x_{2 n}\right)=\frac{\prod_{i, j=1}^{n} G\left(x_{i}, x_{n+j}\right)}{\Delta\left(x_{1}, \ldots, x_{n}\right) \Delta\left(x_{n+1}, \ldots, x_{2 n}\right)} \operatorname{det}_{1 \leq i, j \leq n}\left(\frac{1}{G\left(x_{i}, x_{n+j}\right)}\right),
$$

where

$$
G(x, y)=(\zeta+2) x y(x+y)-\zeta\left(x^{2}+y^{2}\right)-2\left(\zeta^{2}+3 \zeta+1\right) x y+\zeta(2 \zeta+1)(x+y)
$$

Then, $T$ is a symmetric polynomial in $2 n$ variables, depending also as a polynomial on the parameter $\zeta$. Moreover, the space $\Theta_{n}^{\wedge 2 n}$ is spanned by

$$
\prod_{j=1}^{2 n} e^{-2 \pi \mathrm{i} z_{j}} \theta\left(e^{4 \pi \mathrm{i} z_{j}} ; p^{2}\right) \theta\left(p \omega e^{ \pm 2 \pi \mathrm{i} z_{j}} ; p^{2}\right)^{3 n-2} \Delta\left(x_{1}, \ldots, x_{2 n}\right) T\left(x_{1}, \ldots, x_{2 n}\right),
$$

where $x_{j}=x\left(z_{j}\right)$. The function $T$ essentially agrees with the function $H_{n}$ of Zinn-Justin [Z2], see (5.4).

2.4. The functions $T_{n}^{(\mathbf{k})}$. Let $\xi_{j}=x\left(\gamma_{j}\right)$ be the values of $x$ at the half-periods. Explicitly,

$$
\xi_{0}=2 \zeta+1, \quad \xi_{1}=\frac{\zeta}{\zeta+2}, \quad \xi_{2}=\frac{\zeta(2 \zeta+1)}{\zeta+2}, \quad \xi_{3}=1 .
$$

Many functions related to solvable models can be obtained by specializing some variables of the functions $T$ to the values $\xi_{j}$. As a preliminary definition, let

$$
T_{n}^{(\mathbf{k})}\left(x_{1}, \ldots, x_{m}\right)=T\left(x_{1}, \ldots, x_{m}, \boldsymbol{\xi}^{\mathbf{k}}\right),
$$

where $\mathbf{k}=\left(k_{0}, k_{1}, k_{2}, k_{3}\right)$ and

$$
\boldsymbol{\xi}^{\mathbf{k}}=(\underbrace{\xi_{0}, \ldots, \xi_{0}}_{k_{0}}, \underbrace{\xi_{1}, \ldots, \xi_{1}}_{k_{1}}, \underbrace{\xi_{2}, \ldots, \xi_{2}}_{k_{2}}, \underbrace{\xi_{3}, \ldots, \xi_{3}}_{k_{3}}) .
$$

Here, $m$ and $k_{j}$ are non-negative integers restricted by $m+|\mathbf{k}|=2 n$.

We need to relax the condition that $k_{j} \geq 0$. To motivate our extension, note that specializing a variable to $\gamma_{j}$ in the alternant (2.3) leads to a function in $\Theta_{n}^{\wedge(2 n-1)}$ that vanishes if one of the variables equals $\gamma_{j}$. Thus, as a function of each variable, $T_{n}^{(\mathbf{k})}$ uniformizes a function that satisfies (2.1) together with vanishing conditions at $\gamma_{j}$. For instance, if $j=0$, each element in this space has a Taylor expansion of the form

$$
f(z)=\sum_{N=k_{0}}^{\infty} b_{N} z^{2 N+1}
$$

Let $\Phi$ be the $1 / 3$-periodic function $\Phi(z)=\theta\left(e^{ \pm 6 \pi \mathrm{i} z} ; p^{6}\right)^{-k_{0}}$. Using (2.1), it is easy to see that

$$
\Phi(z)\left(f\left(z+\frac{1}{3}\right)+f\left(-z+\frac{1}{3}\right)\right)=\sum_{N=-k_{0}}^{\infty} c_{N} z^{2 N}
$$


This leads to the idea that, to extend (2.10) to $k_{j}<0$, one should impose vanishing conditions at $\xi_{j}$ after first applying the map $(\sigma f)(z)=f(z+1 / 3)+f(-z+1 / 3)$.

It is thus natural to look at the polynomial

$$
\begin{aligned}
& T\left(x_{1}, \ldots, x_{k} ; x_{k+1}, \ldots, x_{2 n}\right) \\
& =\frac{\left(\mathrm{id}^{\otimes k} \otimes \hat{\sigma}^{\otimes(2 n-k)}\right) \Delta\left(x_{1}, \ldots, x_{2 n}\right) T\left(x_{1}, \ldots, x_{2 n}\right)}{\Delta\left(x_{1}, \ldots, x_{k}\right) \Delta\left(x_{k+1}, \ldots, x_{2 n}\right)},
\end{aligned}
$$

where $\hat{\sigma}$ is a uniformization of $\sigma$ (see [R3] for the precise definition). By construction, it is symmetric in its first $k$ and last $2 n-k$ variables. We can obtain different determinant formulas for (2.11) by letting $\hat{\sigma}$ act on different variables in (2.7). Explicitly,

$$
\begin{aligned}
T\left(x_{1}, \ldots, x_{k}, x_{n+1}, \ldots, x_{n+l} ; x_{k+1}, \ldots, x_{n}, x_{n+l+1}, \ldots, x_{2 n}\right) \\
=\frac{\prod_{1 \leq i \leq k, l+1 \leq j \leq n}\left(x_{n+j}-x_{i}\right) \prod_{k+1 \leq i \leq n, 1 \leq j \leq l}\left(x_{i}-x_{n+j}\right) \prod_{i, j=1}^{n} G\left(x_{i}, x_{n+j}\right)}{\Delta\left(x_{1}, \ldots, x_{k}\right) \Delta\left(x_{k+1}, \ldots, x_{n}\right) \Delta\left(x_{n+1}, \ldots, x_{n+l}\right) \Delta\left(x_{n+l+1}, \ldots, x_{2 n}\right)} \\
\quad \times \operatorname{det}_{1 \leq i, j \leq n}\left(B_{i j}\right),
\end{aligned}
$$

where

$$
\begin{aligned}
B_{i, j}= & \begin{cases}\frac{1}{G\left(x_{i}, x_{n+j}\right)}, & 1 \leq i \leq k, 1 \leq j \leq l, \\
\frac{Q\left(x_{i}, x_{n+j}\right)}{\left(x_{n+j}-x_{i}\right) G\left(x_{i}, x_{n+j}\right)}, & 1 \leq i \leq k, l+1 \leq j \leq n, \\
\frac{Q\left(x_{n+j}, x_{i}\right)}{\left(x_{i}-x_{n+j}\right) G\left(x_{i}, x_{n+j}\right)}, & k+1 \leq i \leq n, 1 \leq j \leq l, \\
\frac{R\left(x_{i}, x_{n+j}\right)}{G\left(x_{i}, x_{n+j}\right)}, & k+1 \leq i \leq n, l+1 \leq j \leq n,\end{cases} \\
Q(x, y)= & y(y-2 \zeta-1)((\zeta+2) y-3 \zeta)-x((\zeta+2) y-\zeta)(2 \zeta+1-3 y), \\
R(x, y)= & 3(\zeta+2)^{2} x^{2} y^{2}+\zeta(\zeta+2)(2 \zeta+1)\left(x^{2}+y^{2}\right) \\
& -2\left(\zeta^{2}+4 \zeta+1\right)((\zeta+2) x y+\zeta(2 \zeta+1))(x+y) \\
& 4\left(\zeta^{4}+4 \zeta^{3}+8 \zeta^{2}+4 \zeta+1\right) x y+3 \zeta^{2}(2 \zeta+1)^{2} .
\end{aligned}
$$

As an example, to compute $T\left(x_{1}, x_{2} ; x_{3}, x_{4}\right)$ one may use (2.12) with $k=2$, $l=0$, which gives

$$
\begin{aligned}
& T\left(x_{1}, x_{2} ; x_{3}, x_{4}\right)=\left(\left(x_{4}-x_{1}\right)\left(x_{3}-x_{2}\right) G\left(x_{1}, x_{4}\right) G\left(x_{2}, x_{3}\right) Q\left(x_{1}, x_{3}\right) Q\left(x_{2}, x_{4}\right)\right. \\
& \left.-\left(x_{3}-x_{1}\right)\left(x_{4}-x_{2}\right) G\left(x_{1}, x_{3}\right) G\left(x_{2}, x_{4}\right) Q\left(x_{1}, x_{4}\right) Q\left(x_{2}, x_{3}\right)\right) /\left(x_{2}-x_{1}\right)\left(x_{4}-x_{3}\right)
\end{aligned}
$$


or with $k=l=1$, which gives

$$
\begin{aligned}
T\left(x_{1}, x_{2} ; x_{3}, x_{4}\right)=\left(x_{4}-x_{1}\right)\left(x_{3}-x_{2}\right) & G\left(x_{1}, x_{4}\right) G\left(x_{2}, x_{3}\right) R\left(x_{3}, x_{4}\right) \\
& -G\left(x_{1}, x_{2}\right) G\left(x_{3}, x_{4}\right) Q\left(x_{1}, x_{4}\right) Q\left(x_{2}, x_{3}\right) .
\end{aligned}
$$

To define $T_{n}^{(\mathbf{k})}$ in general, we specialize $k_{j}^{+}=\max \left(k_{j}, 0\right)$ of the left variables and $k_{j}^{-}=\max \left(-k_{j}, 0\right)$ of the right variables to $\xi_{j}$. More precisely, we define

$$
T_{n}^{(\mathbf{k})}\left(x_{1}, \ldots, x_{m}\right)=\frac{(-1)^{\left(\begin{array}{c}
\left|\mathbf{k}^{-}\right| \\
2
\end{array}\right)} T\left(x_{1}, \ldots, x_{m}, \boldsymbol{\xi}^{\mathbf{k}^{+}} ; \boldsymbol{\xi}^{\mathbf{k}^{-}}\right)}{2^{\left|\mathbf{k}^{-}\right|} \prod_{i, j=0}^{3} G\left(\xi_{i}, \xi_{j}\right)^{k_{i}^{-} k_{j}^{+}} \prod_{j=1}^{m} \prod_{i=0}^{3} G\left(x_{j}, \xi_{i}\right)^{k_{i}^{-}}} .
$$

Here, $n \in \mathbb{Z}, \mathbf{k} \in \mathbb{Z}^{4}$ and $m=2 n-|\mathbf{k}| \geq 0$ (we write $|\mathbf{k}|=\sum_{j} k_{j}$ also when some $k_{j}$ are negative). The prefactor in (2.13) has been chosen so that

$$
T_{n}^{(\mathbf{k}+\mathbf{l})}\left(x_{1}, \ldots, x_{m}\right)=T_{n}^{(\mathbf{k})}\left(x_{1}, \ldots, x_{m}, \xi^{\mathbf{l}}\right), \quad \mathbf{l} \in \mathbb{Z}_{\geq 0}^{4} .
$$

To give an example,

$$
\begin{aligned}
T_{0}^{(-2,1,0,0)}(x) & =-\frac{T\left(x, \xi_{1} ; \xi_{0}, \xi_{0}\right)}{4 G\left(\xi_{0}, \xi_{1}\right)^{2} G\left(x, \xi_{0}\right)^{2}}, \\
& =\frac{(2 \zeta+1)^{2}(\zeta+2)}{\zeta^{2} x^{3}}\left(\left(\zeta^{2}+\zeta+1\right) x(2 \zeta+1-x)+\zeta(2 \zeta+1)^{2}\right) .
\end{aligned}
$$

In general, $T_{n}^{(\mathbf{k})}$ is a symmetric rational function, depending also rationally on the parameter $\zeta$. The denominator is elementary and can be explicitly described, so $T_{n}^{(\mathbf{k})}$ is essentially a symmetric polynomial.

In the case $m=0, T_{n}^{(\mathbf{k})}$ depends only on $\zeta$. This case is particularly interesting, as it includes the majority of special cases arising in statistical mechanics. Moreover, these functions can be identified with tau functions of Painlevé VI, see \$4.6. To make it more clear when we are dealing with this case, we write

$$
t^{(\mathbf{k})}=T_{|\mathbf{k}| / 2}^{(\mathbf{k})} .
$$

Here, the only restriction on $\mathbf{k} \in \mathbb{Z}^{4}$ is that $|\mathbf{k}|$ is even.

We can now generalize (2.9). Let $\Theta_{n}^{\mathbf{k}}$ be the space of functions satisfying (2.1), which are analytic except for possible poles at $(1 / 6) \mathbb{Z}+(\tau / 2) \mathbb{Z}$ and such that, for $j=0,1,2,3$,

$$
\lim _{z \rightarrow \gamma_{j}}\left(z-\gamma_{j}\right)^{1-2 k_{j}} f(z)=\lim _{z \rightarrow \gamma_{j}}\left(z-\gamma_{j}\right)^{2}\left(f\left(z+\frac{1}{3}\right)+f\left(-z+\frac{1}{3}\right)\right)=0 .
$$

Then, the function

$$
\begin{aligned}
\prod_{j=1}^{m}\left(e^{-2 \pi \mathrm{i} z_{j}} \theta\left(e^{4 \pi \mathrm{i} z_{j}} ; p^{2}\right) \theta\left(\omega p e^{ \pm 2 \pi \mathrm{i} z_{j}} ; p^{2}\right)^{3 n-2}\right. & \left.\prod_{l=0}^{3}\left(x_{j}-\xi_{l}\right)^{k_{l}}\right) \\
& \times \Delta\left(x_{1}, \ldots, x_{m}\right) T_{n}^{(\mathbf{k})}\left(x_{1}, \ldots, x_{m}\right)
\end{aligned}
$$


is an element of $\left(\Theta_{n}^{\mathbf{k}}\right)^{\wedge m}$.

It is natural to ask whether the space $\Theta_{n}^{\mathbf{k}}$ has dimension $m$. This is a non-trivial question, but we have found that the answer is affirmative. It is easy to see that

$$
\operatorname{dim}\left(\Theta_{n}^{\mathbf{k}}\right)=m \quad \Longleftrightarrow \quad T_{n}^{(\mathbf{k})} \not \equiv 0 .
$$

Thus, the question is settled by the following innocent-looking fact, which is the key for obtaining all our main results. We will comment on the proof in \$2.7. As a consequence, the space $\left(\Theta_{n}^{\mathbf{k}}\right)^{\wedge m}$ is one-dimensional and spanned by (2.14).

Theorem 2.1. The functions $T_{n}^{(\mathbf{k})}$ never vanish identically.

2.5. Modularity. Some of our results are most naturally understood in terms of modular functions. Recall the notation

$$
\begin{aligned}
\Gamma_{0}(n) & =\left\{\left(\begin{array}{ll}
a & b \\
c & d
\end{array}\right) \in \mathrm{SL}(2, \mathbb{Z}) ; c \equiv 0 \bmod n\right\}, \\
\Gamma_{0}(m, n) & =\left\{\left(\begin{array}{ll}
a & b \\
c & d
\end{array}\right) \in \mathrm{SL}(2, \mathbb{Z}) ; c \equiv 0 \bmod m, b \equiv 0 \bmod n\right\} .
\end{aligned}
$$

These groups act on the upper half-plane $\mathbb{H}$ by

$$
\text { A. } \tau=\frac{a \tau+b}{c \tau+d}, \quad A=\left(\begin{array}{ll}
a & b \\
c & d
\end{array}\right) .
$$

A meromorphic function invariant under this action is called a modular function.

The function $\zeta$ is a Hauptmodul for the modular group $\Gamma=\Gamma_{0}(6,2)$, that is, it generates the corresponding field of modular functions. Equivalently, $\tau \mapsto \zeta(2 \tau)$ is a Hauptmodul for the isomorphic group $\Gamma_{0}(12)$. The group $\Gamma$ has six cusps, which correspond to particular limits of $\tau$ in $\mathbb{Q} \cup\{\infty\}$. Denoting by $\overline{\mathbb{H}} / \Gamma$ the union of $\mathbb{H} / \Gamma$ and the cusps, $\zeta$ extends to a bijection from $\overline{\mathbb{H} / \Gamma}$ to $\mathbb{C} \cup\{\infty\}$. The values at the cusps are

$$
\zeta=-2, \quad-1, \quad-\frac{1}{2}, \quad 0, \quad 1, \quad \infty
$$

The normalizer of $\Gamma$ in $\operatorname{SL}(2, \mathbb{Z})$ is $\Gamma_{0}(3)$. Thus, $\Gamma_{0}(3) / \Gamma \simeq \mathrm{S}_{3}$ acts naturally on $\overline{\mathbb{H} / \Gamma}$. The cusps split into two orbits under this action. We will refer to the first orbit, corresponding to $\zeta \in\{-2,-1 / 2,1\}$ as the trigonometric cusps and the other orbit, $\zeta \in\{-1,0, \infty\}$, as the hyperbolic cusps. Our functions $T_{n}^{(\mathbf{k})}$ behave very differently at these two types of cusps.

The cusps are easily understood in the context of the supersymmetric XYZ chain. In homogeneous coordinates, the coupling constants of this chain are

$$
\left(J_{x}: J_{y}: J_{z}\right)=\left(1:-\frac{\zeta}{1+\zeta}: \zeta\right) .
$$

Note that $J_{x} J_{y}+J_{x} J_{z}+J_{y} J_{z}=0$, which is equivalent to $\Delta=-1 / 2$. We see that the trigonometric cusps correspond to the three respective conditions

$$
J_{x}=J_{y}, \quad J_{x}=J_{z}, \quad J_{y}=J_{z}
$$


and thus to the XXZ chain, whereas the hyperbolic cusps correspond to

$$
J_{x}=\infty, \quad J_{y}=\infty, \quad J_{z}=\infty,
$$

that is, to the $\mathrm{XY}$ chain.

2.6. Symmetries. The lattice of polynomials $T_{n}^{(\mathbf{k})}$ has a symmetry under the group $\mathrm{S}_{4}$, acting by rational transformations on the variables $x_{j}$ and $\zeta$ and by permuting the indices $k_{j}$. This can be understood geometrically. Namely, it is generated by an $\mathrm{S}_{2} \times \mathrm{S}_{2}$-action corresponding to simultaneously translating the variables $x_{j}$ by a half-period and an $\mathrm{S}_{3}$-action coming from the modular action of $\Gamma_{0}(3)$. We state the resulting symmetry explicitly for three generators of $\mathrm{S}_{4}$.

Proposition 2.2. The functions $T_{n}^{(\mathbf{k})}=T_{n}^{\left(k_{0}, k_{1}, k_{2}, k_{3}\right)}\left(x_{1}, \ldots, x_{m} ; \zeta\right)$ satisfy

$$
\begin{aligned}
T_{n}^{\left(k_{0}, k_{1}, k_{2}, k_{3}\right)}\left(x_{1}, \ldots, x_{m} ; \zeta\right) \\
=\zeta^{2 n(n-1)} \prod_{j=0}^{3} \xi_{j}^{k_{j}(n-1)} \prod_{j=1}^{m} x_{j}^{n-1} T_{n}^{\left(k_{1}, k_{0}, k_{2}, k_{3}\right)}\left(x_{1}^{-1}, \ldots, x_{m}^{-1} ; \zeta^{-1}\right) \\
=\left(\frac{\zeta-1}{\zeta+2}\right)^{n(n-1)} \\
\quad \times T_{n}^{\left(k_{2}, k_{1}, k_{0}, k_{3}\right)}\left(\frac{(\zeta+2) x_{1}-(1+2 \zeta)}{1-\zeta}, \ldots, \frac{(\zeta+2) x_{m}-(1+2 \zeta)}{1-\zeta} ;-\zeta-1\right) \\
=\left(\frac{\zeta+2}{\zeta(2 \zeta+1)}\right)^{n(n-1)} \prod_{j=0}^{3} \xi_{j}^{k_{j}(n-1)} \prod_{j=1}^{m} x_{j}^{n-1} \\
\quad \times T_{n}^{\left(k_{1}, k_{0}, k_{3}, k_{2}\right)}\left(\frac{\zeta(2 \zeta+1)}{(\zeta+2) x_{1}}, \ldots, \frac{\zeta(2 \zeta+1)}{(\zeta+2) x_{m}} ; \zeta\right) .
\end{aligned}
$$

In the case $m=0$, when we use the notation $t^{(\mathbf{k})}$, there are two additional symmetries not contained in Proposition 2.2. The symmetry in Proposition 2.3 is not hard to understand conceptually (see [R3]), but the one in Proposition 2.4 is more mysterious. It follows from the relation to Painlevé VI, see \$4.6, but it would be interesting to find a more direct proof.

Proposition 2.3. With $n=|\mathbf{k}| / 2$, we have

$$
\begin{aligned}
& t^{\left(k_{0}, k_{1}, k_{2}, k_{3}\right)} \\
& \quad=\frac{(-1)^{n+1}(\zeta+2)^{2\left(k_{1}+k_{2}+n+2\right)} t^{\left(-k_{0}-1,-k_{1}-1,-k_{2}-1,-k_{3}-1\right)}}{12^{n+1} \zeta^{2\left(k_{1}+k_{2}+2 n+3\right)}(\zeta-1)^{2\left(k_{2}+k_{3}+1\right)}(\zeta+1)^{2\left(k_{0}+k_{1}+2 n+3\right)}(2 \zeta+1)^{2\left(k_{0}+k_{2}+1\right)}} .
\end{aligned}
$$

To formulate the last symmetry, let $\left(Y_{k}\right)_{k \in \mathbb{Z}}$ be the solution to the recursion

$$
Y_{k+1} Y_{k-1}=2(2 k+1) Y_{k}^{2}, \quad Y_{0}=Y_{1}=1
$$


that is,

$$
Y_{k}= \begin{cases}\prod_{j=1}^{k} \frac{(2 j-1) !}{(j-1) !}, & k \geq 0 \\ \frac{(-1) \frac{k(k+1)}{2}}{2^{2 k+1}} \prod_{j=1}^{-k-1} \frac{(2 j-1) !}{(j-1) !}, & k<0\end{cases}
$$

Proposition 2.4. With $n=|\mathbf{k}| / 2$ we have

$$
\begin{aligned}
& t^{\left(k_{0}, k_{1}, k_{2}, k_{3}\right)}=(-1)^{\left(k_{0}+k_{1}+n\right)\left(k_{1}+k_{3}+n\right)} \frac{Y_{n-k_{0}} Y_{n-k_{1}} Y_{n-k_{2}} Y_{n-k_{3}}}{Y_{k_{0}} Y_{k_{1}} Y_{k_{2}} Y_{k_{3}}} \\
& \quad \times\left(\frac{\zeta^{k_{1}+k_{2}-n}(\zeta+1)^{k_{0}+k_{1}-n}}{(\zeta-1)^{k_{0}+k_{1}-n}(\zeta+2)^{k_{1}+k_{2}-n}(2 \zeta+1)^{k_{1}+k_{3}-n}}\right)^{n-1} t^{\left(n-k_{0}, n-k_{1}, n-k_{2}, n-k_{3}\right)} .
\end{aligned}
$$

By Proposition 2.3 and Proposition 2.4, the $\mathrm{S}_{4}$ symmetry of Proposition 2.2 is enhanced to an $\mathrm{S}_{4} \times \mathrm{S}_{2} \times \mathrm{S}_{2}$ symmetry when $m=0$. This is natural from the viewpoint of Painlevé theory, see again \$4.6.

2.7. Behaviour at cusps. The proof of the fundamental Theorem 2.1 is based on a careful analysis of the limit of $T_{n}^{(\mathbf{k})}$ as $\zeta \rightarrow-2$ or, equivalently, $p \rightarrow 0$. The behaviour at the other two trigonometric cusps follows using Proposition 2.2.

To indicate what is going on, consider the case when all $k_{j} \geq 0$. Then,

$$
\begin{aligned}
\lim _{\zeta \rightarrow-2}\left(\frac{\zeta+2}{6}\right)^{\left(k_{1}+k_{2}\right)(n-1)-\delta\left(k_{1}+k_{2}-1\right)} T_{n}^{(\mathbf{k})}\left(x_{1}, \ldots, x_{m}\right) & =\left((-1)^{k_{2}} 2^{n} 3^{-k_{1}}\right)^{n-1} \\
\times \chi\left(t_{1}, \ldots, t_{m}\right. & \underbrace{1, \ldots, 1}_{k_{0}}, \underbrace{-1, \ldots,-1}_{k_{3}}) \chi(\underbrace{1, \ldots, 1}_{k_{1}}, \underbrace{-1, \ldots,-1}_{k_{2}}),
\end{aligned}
$$

where $\delta(n)=\left[n^{2} / 4\right]$ and $\chi$ is the symplectic character

$$
\chi\left(t_{1}, \ldots, t_{n}\right)=\chi_{\left[\frac{n-1}{2}\right],\left[\frac{n-2}{2}\right], \ldots, 1,1,0,0}^{\mathfrak{s p}(2 n)}\left(t_{1}, \ldots, t_{n}\right) .
$$

Thus, to prove Theorem 2.1 in this case it is enough to show that (2.18) does not vanish when all variables are specialized to 1 or -1 . Though it would be nice to have a representation-theoretic proof of this fact, our proof is quite computational. We start from the Jacobi-Desnanot identity for the matrix (2.12). In the limit $\zeta \rightarrow-2$, it implies quadratic recurrence relations for the specialized symplectic characters, which can be used to show that they never vanish. This approach extends to the case when some $k_{j}<0$, with the characters replaced by more complicated functions.

We have also investigated the behaviour of $T_{n}^{(\mathbf{k})}$ at the hyperbolic cusps, which is very different. By the symmetries, it is enough to consider the case $\zeta=0$. We have proved that $\lim _{\zeta \rightarrow 0} T_{n}^{(\mathbf{k})} / \zeta^{L}$ exists and is not identically zero, where

$$
L=\left\{\begin{array}{l}
\left(k_{1}+k_{2}\right)\left(2 n-k_{1}-k_{2}-1\right), \\
\left(k_{1}+k_{2}\right)\left(2 n-k_{1}-k_{2}-1\right)+(n+1)\left(n-k_{0}-k_{3}\right), \\
\left(k_{1}+k_{2}\right)\left(2 n-k_{1}-k_{2}-1\right)+(n+1-m)\left(k_{1}+k_{2}-n\right),
\end{array}\right.
$$


when, respectively,

$$
\begin{aligned}
\left|k_{1}+k_{2}+1\right| & \leq m+\left|k_{0}+k_{3}+1\right|, \\
k_{1}+k_{2}+1 & \leq-\left(m+\left|k_{0}+k_{3}+1\right|\right), \\
k_{1}+k_{2}+1 & \geq m+\left|k_{0}+k_{3}+1\right| .
\end{aligned}
$$

This can be used to study the behaviour of related solutions to Painlevé VI at the singular points, see Corollary 4.3 .

2.8. Connection to affine Lie algebras. The character formula for affine Lie algebras is [K, Thm. 10.4]

$$
\operatorname{ch}(\Lambda)=\frac{\sum_{w \in W} \varepsilon(w) e^{w(\Lambda+\rho)-\rho}}{\prod_{\alpha>0}\left(1-e^{-\alpha}\right)^{\operatorname{mult}(\alpha)}} .
$$

Here, the left-hand side is the character of a highest weight module with dominant integral weight $\Lambda$. The sum is over the affine Weyl group and the product is over positive roots. The $\operatorname{sign} \varepsilon$ is the determinant of the Weyl group action, $\rho$ the Weyl vector and $\operatorname{mult}(\alpha)$ the root multiplicity. We refer to $[\mathrm{K}]$ for an explanation of all these terms.

Consider the case of the affine root system $C_{n}^{(1)}$. We denote the long roots of the underlying finite root system $C_{n}$ by $\pm 2 e_{j}$ (in the notation of $[\mathrm{K}], e_{j}=v_{j} / \sqrt{2}$ ). Then, the Cartan algebra $\mathfrak{h}^{*}$ is the complex vector space with basis $\Lambda_{0}, e_{1}, \ldots$, $e_{n}, \delta$. The root sytem $C_{n}^{(1)} \subseteq \mathfrak{h}^{*}$ is the set of non-zero elements of the form $\pm e_{j} \pm e_{k}+m \delta$, where $m \in \mathbb{Z}$. A dominant integral weight is an element of the form

$$
\Lambda=\lambda_{0} \Lambda_{0}+\lambda_{1} e_{1}+\cdots+\lambda_{n} e_{n}+c \delta,
$$

where $c \in \mathbb{C}$ and $\lambda=\left(\lambda_{0}, \ldots, \lambda_{n}\right)$ is a partition, that is, a weakly decreasing sequence of non-negative integers.

Let us write the formal exponential function on $\mathfrak{h}^{*}$ as $e^{\Lambda_{0}}=w, e^{e_{j}}=e^{-2 \pi \mathrm{i} z_{j}}$ and $e^{\delta}=p^{-2}$. Then, the sum and product in (2.19) converge for $|p|<1$. The Weyl group is a semi-direct product of $S_{n},\{ \pm 1\}^{n}$ and $\mathbb{Z}^{n}[\mathrm{~K}, \S 6.5]$. Performing the summation over $\mathbb{Z}^{n}$ and $\{ \pm 1\}^{n}$, one may rewrite (2.19) as

$$
\begin{aligned}
& \operatorname{ch}(\Lambda)= \frac{w^{\Lambda_{0}} p^{-c}\left(p^{4\left(\lambda_{0}+n+1\right)} ; p^{4\left(\lambda_{0}+n+1\right)}\right)_{\infty}^{n}}{\left(p^{2} ; p^{2}\right)_{\infty}^{n} \prod_{j=1}^{n} e^{-2 \pi \mathrm{i} z_{j}} \theta\left(e^{4 \pi \mathrm{i} z_{j}} ; p^{2}\right) \prod_{1 \leq j<k \leq n} e^{-2 \pi \mathrm{i} z_{j}} \theta\left(e^{2 \pi \mathrm{i}\left(z_{j} \pm z_{k}\right)} ; p^{2}\right)} \\
& \times \operatorname{det}_{1 \leq i, j \leq n}\left(e^{-2 \pi \mathrm{i} z_{i}\left(\lambda_{j}+n+1-j\right)} \theta\left(-p^{2\left(\lambda_{0}-\lambda_{j}+j\right)} e^{4 \pi \mathrm{i} z_{i}\left(\lambda_{0}+n+1\right)} ; p^{4\left(\lambda_{0}+n+1\right)}\right)\right. \\
&\left.\quad-e^{2 \pi \mathrm{i} z_{i}\left(\lambda_{j}+n+1-j\right)} \theta\left(-p^{2\left(\lambda_{0}-\lambda_{j}+j\right)} e^{-4 \pi \mathrm{i} z_{i}\left(\lambda_{0}+n+1\right)} ; p^{4\left(\lambda_{0}+n+1\right)}\right)\right) .
\end{aligned}
$$

In particular, since $\operatorname{ch}(0)=1$, the case $\Lambda=0$ gives the determinant form of the $C_{n}^{(1)}$ Macdonald identity [RS].

The determinant in (2.20) agrees with (2.3) if $n$ is replaced by $2 n$ and

$$
\lambda=(n-1, n-1, n-1, n-2, n-2, \ldots, 1,1,0,0) .
$$


(This may be compared with (2.5) and (2.18).) We conclude that, for some multiplier $C_{n}(\tau)$,

$$
\prod_{j=1}^{2 n} \theta\left(p \omega e^{ \pm 2 \pi \mathrm{i} z_{j}} ; p^{2}\right)^{n-1} T\left(x_{1}, \ldots, x_{2 n}\right)=C_{n}(\tau) p^{c} w^{1-n} \operatorname{ch}(\Lambda),
$$

where $\operatorname{ch}(\Lambda)$ is an affine Lie algebra character of type $C_{2 n}^{(1)}$.

There are also close relations to other affine Lie algebras. For instance, in the context of the three-colour model, the fundamental object is $T_{n}^{(0,0,0,-1)}$ rather than $T=T_{n}^{(0,0,0,0)}$, see [R2] and \$5.1. This function can in a similar way be identified with a character of type $A_{2 n+1}^{(2)}$. We plan to treat this topic in more detail in the near future.

\section{SCHRÖDINGER EQUATION}

3.1. Elliptic Schrödinger equation. Any element in the one-dimensional space $\left(\Theta_{n}^{(\mathbf{k})}\right)^{\wedge m}$ satisfies a Schrödinger equation with elliptic potential.

Theorem 3.1. Let $\Psi\left(z_{1}, \ldots, z_{m}, \tau\right)$ be a meromorphic function, which for fixed $\tau$ belongs to $\left(\Theta_{n}^{\mathbf{k}}\right)^{\wedge m}$, and let

$$
\begin{aligned}
\Phi=\prod_{j=1}^{m}\left(\left(e^{-3 \pi \mathrm{i} z_{j}} \theta\left(e^{6 \pi \mathrm{i} z_{j}} ; p^{6}\right)\right)^{k_{0}}\right. & \theta\left(p^{3} e^{6 \pi \mathrm{i} z_{j}} ; p^{6}\right)^{k_{1}} \\
& \left.\times \theta\left(-p^{3} e^{6 \pi \mathrm{i} z_{j}} ; p^{6}\right)^{k_{2}}\left(e^{-3 \pi \mathrm{i} z_{j}} \theta\left(-e^{6 \pi \mathrm{i} z_{j}} ; p^{6}\right)\right)^{k_{3}}\right) .
\end{aligned}
$$

Then,

$$
\mathcal{H} \Phi^{-1} \Psi=C \Phi^{-1} \Psi
$$

where, writing $z_{j}=x_{j} / 3, \tau=2 \pi \mathrm{i} t / 3$,

$$
\mathcal{H}=-m \frac{\partial}{\partial t}+\sum_{j=1}^{m}\left(\frac{1}{2} \frac{\partial^{2}}{\partial x_{j}^{2}}-V\left(x_{j}\right)\right),
$$

$C$ is independent of the variables $z_{j}$ and $V$ is the potential (1.2).

The variables $x_{j}$ and $t$ are only used here to write (3.1) in the form it usually appears in the literature; they are not related to variables $x_{j}$ and $t$ used elsewhere. Note that $\Psi$ is uniquely determined up to a factor depending on $\tau$; the factor $C$ depends on this choice of normalization. In particular, if $C=0$ and $m=1$ we recover (1.1).

Theorem 3.1 follows from the fact that $\left(\Theta_{n}^{\mathbf{k}}\right)^{\wedge m}$ is one-dimensional, which is a consequence of Theorem 2.1. It is thus enough to prove that this space is preserved by $\Phi \mathcal{H} \Phi^{-1}$, which is a straight-forward exercise.

The following result is a uniformized version of Theorem 3.1. The special case $m=1, \mathbf{k}=(0, n, n,-1)$, is equivalent to [BM1, Eq. (27)] (given there without a complete proof, since it was not known at the time that $\operatorname{dim} \Theta_{n}^{(0, n, n,-1)}=1$ ). 
The case $m=1, \mathbf{k}=(n, n, 0,-1)$ was conjectured in $[\mathrm{MB}]$; it is in fact equivalent to the case $\mathbf{k}=(0, n, n,-1)$ by Proposition 2.2. Moreover, the case $m=2 n$, $\mathbf{k}=(0,0,0,0)$ is equivalent to [Z2, Eq. (50)].

Theorem 3.2. The function $T_{n}^{(\mathbf{k})}$ satisfies an algebraic differential equation

$$
\begin{aligned}
\left(\sum_{j=1}^{m}\left(a\left(x_{j}, \zeta\right) \frac{\partial^{2}}{\partial x_{j}^{2}}+b\left(x_{j}, \zeta\right) \frac{\partial}{\partial x_{j}}+c\left(x_{j}, \zeta\right)\right)\right. & \left.+d(\zeta) \frac{\partial}{\partial \zeta}+e(\zeta)\right) \\
\Delta\left(x_{1}, \ldots, x_{m}\right) T_{n}^{(\mathbf{k})}\left(x_{1}, \ldots, x_{m}\right) & =0 .
\end{aligned}
$$

Here,

$$
\begin{aligned}
a(x, \zeta) & =(x-2 \zeta-1)(x-1)((\zeta+2) x-\zeta)((\zeta+2) x-\zeta(2 \zeta+1)) \\
d(\zeta) & =2 m \zeta(\zeta-1)(\zeta+1)(\zeta+2)(2 \zeta+1) .
\end{aligned}
$$

We refer to [R4] for explicit expressions for the rational functions $b, c$ and $e$.

Making an appropriate change of variables in Theorem 3.1, it is in principle straight-forward to show that (3.2) holds up to a change of the constant term $e(\zeta)$. To compute $e(\zeta)$ is not trivial, see [R4, §3.3], but it is crucial for obtaining the relation to the Painlevé VI equation described in 4 .

3.2. An application. Theorem 3.2 can be used to derive many bilinear identities for the functions $t^{(\mathbf{k})}$. An alternative way to derive such identities is to use the identification with Painlevé tau functions discussed in \$4. We illustrate the first method by the following result, which aims at characterizing $t^{(\mathbf{k})}$ by a very short list of properties. This will in fact be used in $\$ 4$ to obtain the above-mentioned identification with tau functions.

Theorem 3.3. The functions $t^{(\mathbf{k})}$ satisfy the two identities

$$
\begin{aligned}
& t^{\left(\mathbf{k}-2 \mathbf{e}_{0}\right)} t^{\left(\mathbf{k}+\mathbf{e}_{0}+\mathbf{e}_{1}\right)}=\zeta^{2}(\zeta+1)(\zeta-1)(2 \zeta+1)^{2} \\
& \times\left(\frac{1}{2 k_{0}-1} t^{(\mathbf{k})} \frac{d t^{\left(\mathbf{k}-\mathbf{e}_{0}+\mathbf{e}_{1}\right)}}{d \zeta}-\frac{1}{2 k_{0}+1} \frac{d t^{(\mathbf{k})}}{d \zeta} t^{\left(\mathbf{k}-\mathbf{e}_{0}+\mathbf{e}_{1}\right)}\right) \\
&+\frac{\zeta(2 \zeta+1)}{2\left(2 k_{0}-1\right)\left(2 k_{0}+1\right)(\zeta+2)} A^{(\mathbf{k})} t^{(\mathbf{k})} t^{\left(\mathbf{k}-\mathbf{e}_{0}+\mathbf{e}_{1}\right)}, \\
& t^{\left(\mathbf{k}-2 \mathbf{e}_{0}\right)} t^{\left(\mathbf{k}+\mathbf{e}_{0}-\mathbf{e}_{1}\right)}=\frac{(\zeta+1)(\zeta-1)(2 \zeta+1)^{2}(\zeta+2)^{2}}{\zeta^{2}} \\
& \times\left(\frac{1}{2 k_{0}-1} t^{(\mathbf{k})} \frac{d t^{\left(\mathbf{k}-\mathbf{e}_{0}-\mathbf{e}_{1}\right)}}{d \zeta}-\frac{1}{2 k_{0}+1} \frac{d t^{(\mathbf{k})}}{d \zeta} t^{\left(\mathbf{k}-\mathbf{e}_{0}-\mathbf{e}_{1}\right)}\right) \\
&+\frac{(2 \zeta+1)(\zeta+2)}{2\left(2 k_{0}-1\right)\left(2 k_{0}+1\right) \zeta^{3}} B^{(\mathbf{k})} t^{(\mathbf{k})} t^{\left(\mathbf{k}-\mathbf{e}_{0}-\mathbf{e}_{1}\right)},
\end{aligned}
$$


where $\mathbf{e}_{j}$ are unit vectors and

$$
\begin{aligned}
A^{(\mathbf{k})}= & \left(2 \zeta^{4}-23 \zeta^{3}-36 \zeta^{2}-5 \zeta+8\right) k_{0}^{2}-\zeta(2 \zeta+1)\left(3 \zeta^{2}+10 \zeta+5\right) k_{1}\left(2 k_{0}+k_{1}\right) \\
& -\zeta\left(6 \zeta^{3}+19 \zeta^{2}+4 \zeta-11\right) k_{2}^{2}-\zeta(2 \zeta+1)\left(3 \zeta^{2}+2 \zeta+1\right) k_{3}^{2} \\
& -2 \zeta(\zeta-1)(2 \zeta+1)(\zeta+3)\left(k_{0}+k_{1}\right) k_{2} \\
& -2(\zeta-1)(2 \zeta+1)\left(3 \zeta^{2}+9 \zeta+4\right)\left(k_{0}+k_{1}\right) k_{3} \\
& -2(2 \zeta+1)\left(\zeta^{3}+6 \zeta^{2}+3 \zeta-4\right) k_{2} k_{3}-4(2 \zeta+1)\left(\zeta^{2}+5 \zeta+3\right)\left(k_{0}+k_{1}\right) \\
& +4(2 \zeta+1)\left(2 \zeta^{3}+5 \zeta^{2}-\zeta-3\right) k_{2}+4(2 \zeta+1)\left(\zeta^{2}+\zeta+1\right) k_{3} \\
& -4(\zeta+1)^{2}\left(2 \zeta^{2}-\zeta+2\right), \\
B^{(\mathbf{k})}= & \left(10 \zeta^{4}+13 \zeta^{3}-28 \zeta^{2}-41 \zeta-8\right) k_{0}^{2}-\zeta(2 \zeta+1)\left(3 \zeta^{2}+10 \zeta+5\right) k_{1}\left(k_{1}-2 k_{0}\right) \\
& -\zeta\left(6 \zeta^{3}+19 \zeta^{2}+4 \zeta-11\right) k_{2}^{2}-\zeta(2 \zeta+1)\left(3 \zeta^{2}+2 \zeta+1\right) k_{3}^{2} \\
& +2 \zeta(\zeta-1)(2 \zeta+1)(\zeta+3)\left(k_{0}-k_{1}\right) k_{2}-2(2 \zeta+1)\left(\zeta^{3}+6 \zeta^{2}+3 \zeta-4\right) k_{2} k_{3} \\
& +2(\zeta-1)(2 \zeta+1)\left(3 \zeta^{2}+9 \zeta+4\right)\left(k_{0}-k_{1}\right) k_{3} \\
& +2(\zeta-1)(2 \zeta+1)(\zeta+3)(3 \zeta+2)\left(k_{1}-k_{0}\right) \\
& +2(2 \zeta+1)\left(5 \zeta^{3}+12 \zeta^{2}-5 \zeta-6\right) k_{2}+2(2 \zeta+1)\left(3 \zeta^{3}+8 \zeta^{2}-3 \zeta-2\right) k_{3} \\
& -2\left(8 \zeta^{4}+18 \zeta^{3}-7 \zeta^{2}-18 \zeta-4\right) .
\end{aligned}
$$

Moreover, the lattice of functions $t^{(\mathbf{k})}$, where $\mathbf{k} \in \mathbb{Z}^{4}$ with $\sum_{j} k_{j}$ even, is uniquely determined by (3.3), the three values

$$
t^{(0,0,0,0)}=t^{(1,-1,0,0)}=1, \quad t^{(0,-1,-1,0)}=-\frac{2 \zeta^{2}(\zeta-1)(\zeta+1)^{2}(2 \zeta+1)}{(\zeta+2)^{2}}
$$

and the symmetries of Proposition 2.2 (with $m=0$ ) and Proposition 2.3 .

To explain the main idea, we sketch the proof of (3.3a). Applying the JacobiDesnanot identity to the matrix (2.12) yields the recursion

$$
\begin{aligned}
(a-b)(c-d) T(\mathbf{x} ; \mathbf{y}) T(a, b, c, d, \mathbf{x} ; \mathbf{y}) & =G(a, d) G(b, c) T(a, c, \mathbf{x} ; \mathbf{y}) T(b, d, \mathbf{x} ; \mathbf{y}) \\
& -G(a, c) G(b, d) T(a, d, \mathbf{x} ; \mathbf{y}) T(b, c, \mathbf{x} ; \mathbf{y})
\end{aligned}
$$

An appropriate specialization of the variables gives

$$
\begin{aligned}
& \left(x-\xi_{0}\right)\left(\xi_{0}-\xi_{1}\right) t^{(\mathbf{k})} T_{n+2}^{\left(\mathbf{k}+2 \mathbf{e}_{0}+\mathbf{e}_{1}\right)}(x) \\
& \quad=G\left(x, \xi_{1}\right) G\left(\xi_{0}, \xi_{0}\right) T_{n+1}^{\left(\mathbf{k}+\mathbf{e}_{0}\right)}(x) t^{\left(\mathbf{k}+\mathbf{e}_{0}+\mathbf{e}_{1}\right)}-G\left(x, \xi_{0}\right) G\left(\xi_{0}, \xi_{1}\right) t^{\left(\mathbf{k}+2 \mathbf{e}_{0}\right)} T_{n+1}^{\left(\mathbf{k}+\mathbf{e}_{1}\right)}(x),
\end{aligned}
$$


where $|\mathbf{k}|=2 n$. Differentiating with respect to $x$ and letting $x=\xi_{0}$ gives

$$
\begin{aligned}
& \left(\xi_{0}-\xi_{1}\right) t^{(\mathbf{k})} t^{\left(\mathbf{k}+3 \mathbf{e}_{0}+\mathbf{e}_{1}\right)} \\
& =\left(G\left(\xi_{0}, \xi_{0}\right) \frac{\partial G}{\partial x}\left(\xi_{0}, \xi_{1}\right)-\frac{\partial G}{\partial x}\left(\xi_{0}, \xi_{0}\right) G\left(\xi_{0}, \xi_{1}\right)\right) t^{\left(\mathbf{k}+2 \mathbf{e}_{0}\right)} t^{\left(\mathbf{k}+\mathbf{e}_{0}+\mathbf{e}_{1}\right)} \\
& +G\left(\xi_{0}, \xi_{0}\right) G\left(\xi_{0}, \xi_{1}\right)\left(\frac{\partial T_{n+1}^{\left(\mathbf{k}+\mathbf{e}_{0}\right)}}{\partial x}\left(\xi_{0}\right) t^{\left(\mathbf{k}+\mathbf{e}_{0}+\mathbf{e}_{1}\right)}-t^{\left(\mathbf{k}+2 \mathbf{e}_{0}\right)} \frac{\partial T_{n+1}^{\left(\mathbf{k}+\mathbf{e}_{1}\right)}}{\partial x}\left(\xi_{0}\right)\right) .
\end{aligned}
$$

The point is now that the specialized derivatives of $T$-functions can be expressed in terms of $t$-functions using the Schrödinger equation. Indeed, since $a\left(\xi_{0}, \zeta\right)=0$, if we let $m=1$ and $x_{1}=\xi_{0}$ in (3.2), we get a linear relation of the form

$$
A \frac{\partial T_{n}^{(\mathbf{k})}}{\partial x}\left(\xi_{0}\right)+B T_{n}^{(\mathbf{k})}\left(\xi_{0}\right)+C \frac{\partial T_{n}^{(\mathbf{k})}}{\partial \zeta}\left(\xi_{0}\right)=0
$$

On the other hand, differentiating the equality $T_{n}^{(\mathbf{k})}\left(\xi_{0}\right)=t^{\left(\mathbf{k}+\mathbf{e}_{0}\right)}$ gives

$$
2 \frac{\partial T_{n}^{(\mathbf{k})}}{\partial x}\left(\xi_{0}\right)+\frac{\partial T_{n}^{(\mathbf{k})}}{\partial \zeta}\left(\xi_{0}\right)=\frac{d t^{\left(\mathbf{k}+\mathbf{e}_{0}\right)}}{d \zeta} .
$$

Eliminating $\partial T_{n}^{(\mathbf{k})} / \partial \zeta$ from these two equations gives

$$
\frac{\partial T_{n}^{(\mathbf{k})}}{\partial x}\left(\xi_{0}\right)=D t^{\left(\mathbf{k}+\mathbf{e}_{0}\right)}+E \frac{d t^{\left(\mathbf{k}+e_{0}\right)}}{d \zeta},
$$

where $D$ and $E$ are explicit coefficients. Using (3.5) on the right-hand side of (3.4) gives, after replacing $k_{0}$ by $k_{0}-2$, (3.3a).

\section{Painlevé VI}

4.1. Bäcklund transformations. Painlevé VI is the differential equation

$$
\begin{aligned}
\frac{d^{2} q}{d t^{2}}= & \frac{1}{2}\left(\frac{1}{q}+\frac{1}{q-1}+\frac{1}{q-t}\right)\left(\frac{d q}{d t}\right)^{2}-\left(\frac{1}{t}+\frac{1}{t-1}+\frac{1}{q-t}\right) \frac{d q}{d t} \\
& +\frac{q(q-1)(q-t)}{t^{2}(t-1)^{2}}\left(\alpha+\beta \frac{t}{q^{2}}+\gamma \frac{t-1}{(q-1)^{2}}+\delta \frac{t(t-1)}{(q-t)^{2}}\right) .
\end{aligned}
$$

We will briefly review the rich symmetry theory of this equation. It is mainly due to Okamoto [Ok], but we follow the exposition of Noumi and Yamada [NY]. We introduce parameters $\alpha_{0}, \ldots, \alpha_{4}$ satisfying the constraint

$$
\alpha_{0}+\alpha_{1}+2 \alpha_{2}+\alpha_{3}+\alpha_{4}=1
$$

and related to the parameters of (4.1) by

$$
\alpha=\frac{\alpha_{1}^{2}}{2}, \quad \beta=-\frac{\alpha_{4}^{2}}{2}, \quad \gamma=\frac{\alpha_{3}^{2}}{2}, \quad \delta=\frac{1-\alpha_{0}^{2}}{2} .
$$


We let

$$
\begin{aligned}
H=q(q-1)(q-t) p^{2}-\left\{\left(\alpha_{0}-1\right) q(q-1)+\alpha_{3} q(q-t)\right. & \left.+\alpha_{4}(q-1)(q-t)\right\} p \\
& +\alpha_{2}\left(\alpha_{1}+\alpha_{2}\right)(q-t) .
\end{aligned}
$$

Then, (4.1) is equivalent to the Hamiltonian system

$$
t(t-1) \frac{d q}{d t}=\frac{\partial H}{\partial p}, \quad t(t-1) \frac{d p}{d t}=-\frac{\partial H}{\partial q} .
$$

The system (4.3) admits many symmetries, or Bäcklund transformations. Indeed, it is invariant under the involutions $s_{j}, r_{j}$ and $t_{j}$ defined as follows.

\begin{tabular}{|c||ccccc|ccc|}
\hline & $\alpha_{0}$ & $\alpha_{1}$ & $\alpha_{2}$ & $\alpha_{3}$ & $\alpha_{4}$ & $q$ & $p$ & $t$ \\
\hline \hline$s_{0}$ & $-\alpha_{0}$ & $\alpha_{1}$ & $\alpha_{2}+\alpha_{0}$ & $\alpha_{3}$ & $\alpha_{4}$ & $q$ & $p-\frac{\alpha_{0}}{q-t}$ & $t$ \\
$s_{1}$ & $\alpha_{0}$ & $-\alpha_{1}$ & $\alpha_{2}+\alpha_{1}$ & $\alpha_{3}$ & $\alpha_{4}$ & $q$ & $p$ & $t$ \\
$s_{2}$ & $\alpha_{0}+\alpha_{2}$ & $\alpha_{1}+\alpha_{2}$ & $-\alpha_{2}$ & $\alpha_{3}+\alpha_{2}$ & $\alpha_{4}+\alpha_{2}$ & $q+\frac{\alpha_{2}}{p}$ & $p$ & $t$ \\
$s_{3}$ & $\alpha_{0}$ & $\alpha_{1}$ & $\alpha_{2}+\alpha_{3}$ & $-\alpha_{3}$ & $\alpha_{4}$ & $q$ & $p-\frac{\alpha_{3}}{q-1}$ & $t$ \\
$s_{4}$ & $\alpha_{0}$ & $\alpha_{1}$ & $\alpha_{2}+\alpha_{4}$ & $\alpha_{3}$ & $-\alpha_{4}$ & $q$ & $p-\frac{\alpha_{4}}{q}$ & $t$ \\
$r_{1}$ & $\alpha_{1}$ & $\alpha_{0}$ & $\alpha_{2}$ & $\alpha_{4}$ & $\alpha_{3}$ & $\frac{t(q-1)}{q-t}$ & $\frac{(t-q)\left((q-t) p+\alpha_{2}\right)}{t(t-1)}$ & $t$ \\
$r_{3}$ & $\alpha_{3}$ & $\alpha_{4}$ & $\alpha_{2}$ & $\alpha_{0}$ & $\alpha_{1}$ & $\frac{t}{q}$ & $-\frac{q\left(p q+\alpha_{2}\right)}{t}$ & $t$ \\
$t_{1}$ & $\alpha_{0}$ & $\alpha_{4}$ & $\alpha_{2}$ & $\alpha_{3}$ & $\alpha_{1}$ & $\frac{1}{q}$ & $-q\left(p q+\alpha_{2}\right)$ & $\frac{1}{t}$ \\
$t_{3}$ & $\alpha_{0}$ & $\alpha_{1}$ & $\alpha_{2}$ & $\alpha_{4}$ & $\alpha_{3}$ & $1-q$ & $-p$ & $1-t$ \\
\hline
\end{tabular}

We will write $r_{4}=r_{1} r_{3}=r_{3} r_{1}$. We consider the Bäcklund transformations as automorphisms of the differential field $\mathcal{F}_{0}$ generated by $\alpha_{j}, q, p$ and $t$, subject to the relation (4.2), and equipped with the derivation

$$
\delta=\frac{\partial H}{\partial p} \frac{\partial}{\partial q}-\frac{\partial H}{\partial q} \frac{\partial}{\partial p}+t(t-1) \frac{\partial}{\partial t} .
$$

The transformations

$$
\begin{array}{ll}
T_{1}=r_{1} s_{1} s_{2} s_{3} s_{4} s_{2} s_{1}, & T_{2}=s_{0} s_{2} s_{1} s_{3} s_{4} s_{2} s_{1} s_{3} s_{4} s_{2}, \\
T_{3}=r_{3} s_{3} s_{2} s_{1} s_{4} s_{2} s_{3}, & T_{4}=r_{4} s_{4} s_{2} s_{1} s_{3} s_{2} s_{4}
\end{array}
$$

generate an action of $\mathbb{Z}^{4}$ on $\mathcal{F}_{0}$.

4.2. Tau functions. A rational solution of Painlevé VI can be identified with a field automorphism $\mathbf{X}: \mathcal{F}_{0} \rightarrow \mathbb{C}(t)$ such that $\mathbf{X}(t)=t$ and

$$
\mathbf{X} \circ \delta=\delta \circ \mathbf{X} \text {, }
$$

where $\delta$ acts on $\mathbb{C}(t)$ as $t(t-1) \cdot d / d t$. Since we are interested in more general algebraic solutions, we will define extensions $\mathcal{F}$ and $\mathcal{M}$ of the differential fields $\mathcal{F}_{0}$ and $\mathbb{C}(t)$, respectively, and consider solutions as automorphisms $\mathbf{X}: \mathcal{F} \rightarrow \mathcal{M}$. We need $\mathcal{F}$ to contain tau functions, which represent inverse logarithmic derivatives of appropriate modifications of the Hamiltonian. One way to do this was proposed 
by Masuda $[\mathrm{M}]$. It is, however, not ideal for our purposes and we will therefore work with a variation of Masuda's construction.

We introduce the modified Hamiltonian

$$
\begin{aligned}
h_{0}= & H+\frac{t}{12}\left(2\left(\alpha_{0}-1\right)^{2}-\alpha_{1}^{2}+2 \alpha_{3}^{2}-\alpha_{4}^{2}+6\left(\alpha_{0}-1\right) \alpha_{3}\right) \\
& +\frac{t-1}{12}\left(2\left(\alpha_{0}-1\right)^{2}-\alpha_{1}^{2}-\alpha_{3}^{2}+2 \alpha_{4}^{2}+6\left(\alpha_{0}-1\right) \alpha_{4}\right) .
\end{aligned}
$$

Note that (4.3) holds with $H$ replaced by $h_{0}$. We also define

$$
h_{1}=r_{1}\left(h_{0}\right), \quad h_{3}=r_{3}\left(h_{0}\right), \quad h_{4}=r_{4}\left(h_{0}\right), \quad h_{2}=h_{1}+s_{1}\left(h_{1}\right)-\frac{t}{3}+\frac{1}{6} .
$$

We define $\mathcal{F}$ to be the field extension of $\mathcal{F}_{0}$ by the additional generators $u, v$, $\tau_{0}, \ldots, \tau_{4}$. The generators $u$ and $v$ satisfy

$$
t=u^{2} v^{4}, \quad 1-t=u^{4} v^{2}
$$

and thus formally correspond to the roots $t^{-1 / 6}(1-t)^{1 / 3}$ and $t^{1 / 3}(1-t)^{-1 / 6}$. We extend $\delta$ to the new generators by

$$
\delta(u)=\frac{u(t+1)}{6}, \quad \delta(v)=\frac{v(t-2)}{6}, \quad \delta\left(\tau_{j}\right)=\tau_{j} h_{j}, \quad j=0, \ldots, 4 .
$$

Finally, we extend the Bäcklund transformations to $\mathcal{F}$ by the following table.

\begin{tabular}{|c||cc|ccccc|}
\hline & $u$ & $v$ & $\tau_{0}$ & $\tau_{1}$ & $\tau_{2}$ & $\tau_{3}$ & $\tau_{4}$ \\
\hline \hline$s_{0}$ & $u$ & $v$ & $\frac{\mathrm{i}(t-q) \tau_{2}}{u^{2} v^{2} \tau_{0}}$ & $\tau_{1}$ & $\tau_{2}$ & $\tau_{3}$ & $\tau_{4}$ \\
$s_{1}$ & $u$ & $v$ & $\tau_{0}$ & $\frac{\mathrm{i} u v \tau_{2}}{\tau_{1}}$ & $\tau_{2}$ & $\tau_{3}$ & $\tau_{4}$ \\
$s_{2}$ & $u$ & $v$ & $\tau_{0}$ & $\tau_{1}$ & $\frac{p \tau_{0} \tau_{1} \tau_{3} \tau_{4}}{\tau_{2}}$ & $\tau_{3}$ & $\tau_{4}$ \\
$s_{3}$ & $u$ & $v$ & $\tau_{0}$ & $\tau_{1}$ & $\tau_{2}$ & $\frac{(1-q) \tau_{2}}{u \tau_{3}}$ & $\tau_{4}$ \\
$s_{4}$ & $u$ & $v$ & $\tau_{0}$ & $\tau_{1}$ & $\tau_{2}$ & $\tau_{3}$ & $\frac{q \tau_{2}}{v \tau_{4}}$ \\
$r_{1}$ & $u$ & $-v$ & $\tau_{1}$ & $\tau_{0}$ & $\frac{(q-t) \tau_{2}}{u^{3} v^{3}}$ & $\tau_{4}$ & $\tau_{3}$ \\
$r_{3}$ & $-u$ & $v$ & $\tau_{3}$ & $\tau_{4}$ & $\frac{i q \tau_{2}}{u v^{2}}$ & $\tau_{0}$ & $\tau_{1}$ \\
$t_{1}$ & $u$ & $\frac{\mathrm{i}}{u v}$ & $\mathrm{i} \tau_{0}$ & $\tau_{4}$ & $-q \tau_{2}$ & $\tau_{3}$ & $\tau_{1}$ \\
$t_{3}$ & $v$ & $u$ & $\mathrm{i} \tau_{0}$ & $\tau_{1}$ & $\tau_{2}$ & $\tau_{4}$ & $\tau_{3}$ \\
\hline
\end{tabular}

With this definition, the operators $T_{j}$ define an action of $\mathbb{Z}^{4}$ on $\mathcal{F}$. This is in contrast to the alternative definition of Masuda. However, not all algebraic relations for Bäcklund transformations extend from $\mathcal{F}_{0}$ to $\mathcal{F}$. For instance, $t_{1}$ and $t_{3}$ generate an action of the dicyclic group of order 12 on $\mathcal{F}$, but an action of $\mathrm{S}_{3}$ on $\mathcal{F}_{0}$.

An important application of tau functions is encoded in the identity

$$
T_{1}^{l_{1}} T_{2}^{l_{2}} T_{3}^{l_{3}} T_{4}^{l_{4}}(q)=(-1)^{l_{3}+l_{4}} \mathrm{i} u v^{2} \frac{\tau_{l_{1}, l_{2}, l_{3}, l_{4}+1} \tau_{l_{1}, l_{2}+1, l_{3}, l_{4}-1}}{\tau_{l_{1}+1, l_{2}, l_{3}, l_{4}} \tau_{l_{1}-1, l_{2}+1, l_{3}, l_{4}}}
$$

where

$$
\tau_{l_{1} l_{2} l_{3} l_{4}}=T_{1}^{l_{1}} T_{2}^{l_{2}} T_{3}^{l_{3}} T_{4}^{l_{4}} \tau_{0} .
$$


Thus, if we act on a solution of Painlevé VI by $\mathbb{Z}^{4}$, the resulting new solutions will typically factor into four non-trivial parts, see (4.18) for an example.

Painlevé VI can be reformulated as a differential equation for the Hamiltonian, known as the $\mathrm{E}_{\mathrm{VI}}$ equation [JM, $\left.\mathrm{Ok}\right]$. In terms of the parameters

$$
b_{1}=\frac{\alpha_{3}+\alpha_{4}}{2}, \quad b_{2}=\frac{\alpha_{4}-\alpha_{3}}{2}, \quad b_{3}=\frac{\alpha_{0}+\alpha_{1}-1}{2}, \quad b_{4}=\frac{\alpha_{0}-\alpha_{1}-1}{2}
$$

it takes the form

$$
\begin{aligned}
\frac{d h}{d t}\left(t(t-1) \frac{d^{2} h}{d t^{2}}\right)^{2}+\left(\frac{d h}{d t}\left(2 h-(2 t-1) \frac{d h}{d t}\right)+b_{1} b_{2} b_{3} b_{4}\right)^{2} & =\prod_{k=1}^{4}\left(\frac{d h}{d t}+b_{k}^{2}\right),
\end{aligned}
$$

where $h$ is related to (4.5) by

$$
h=h_{0}-\frac{C}{24}(2 t-1)
$$

with

$$
C=\left(\alpha_{0}-1\right)^{2}+\alpha_{1}^{2}+\alpha_{3}^{2}+\alpha_{4}^{2}=2\left(b_{1}^{2}+b_{2}^{2}+b_{3}^{2}+b_{4}^{2}\right) .
$$

Expressing $h$ in terms of tau functions, (4.8) takes a rather complicated form. To obtain a simpler identity, we first cancel the term $\prod_{k} b_{k}^{2}$ and the factor $d h / d t$ on both sides, then differentiate in $t$ and finally cancel the factor $d^{2} h / d t^{2}$. Rewriting the result in terms of $\tau=\tau_{0}$ gives

$$
\begin{gathered}
\delta^{4}(\tau) \tau-4 \delta^{3}(\tau) \delta(\tau)+2(1-2 t) \delta^{3}(\tau) \tau+3 \delta^{2}(\tau)^{2}-2(1-2 t) \delta^{2}(\tau) \delta(\tau) \\
-\frac{(C-6) t(t-1)+C-3}{3} \delta^{2}(\tau) \tau+\frac{C\left(t^{2}-t+1\right)-3}{3} \delta(\tau)^{2} \\
+\frac{C t(t-1)(2 t-1)}{6} \delta(\tau) \tau-\frac{t(t-1) G}{8} \tau^{2}=0,
\end{gathered}
$$

where

$$
\begin{aligned}
G=\left(\alpha_{4}-\alpha_{3}\right)\left(\alpha_{3}+\alpha_{4}\right) & \left(\alpha_{0}+\alpha_{1}-1\right)\left(\alpha_{0}-\alpha_{1}-1\right) t \\
& +\left(\alpha_{3}-\alpha_{1}\right)\left(\alpha_{3}+\alpha_{1}\right)\left(\alpha_{0}+\alpha_{4}-1\right)\left(\alpha_{0}-\alpha_{4}-1\right) .
\end{aligned}
$$

Acting on this equation by $\mathbb{Z}^{4}$ we find that $\tau=\tau_{l_{1} l_{2} l_{3} l_{4}}$ satisfies (4.10), with $C$ and $G$ obtained from (4.9) and (4.11) by replacing each $\alpha_{j}$ with $\alpha_{j}-l_{j}$. Here, $l_{0}$ is defined by

$$
l_{0}+l_{1}+2 l_{2}+l_{3}+l_{4}=0 .
$$

Somewhat surprisingly, we have not found this result in the literature. Analogous results for other Painlevé equations are discussed in [C]. 


\subsection{An algebraic Picard solution. When}

$$
\alpha_{0}=\alpha_{1}=\alpha_{3}=\alpha_{4}=0, \quad \alpha_{2}=\frac{1}{2},
$$

Painlevé VI can be solved explicitly in terms of Weierstrass's $\wp$-function. This was done by Picard already in $1889[\mathrm{P}]$. The general solution is labelled by two complex parameters $\nu_{1}, \nu_{2}$; it is algebraic if $\nu_{1}, \nu_{2} \in \mathbb{Q}$ [Ma]. In [BM2], the solution with $\left(\nu_{1}, \nu_{2}\right)=(1,1 / 3)$ was expressed as

$$
q^{4}-4 t q^{3}+6 t q^{2}-4 t q+t^{2}=0 .
$$

Considering this as a seed solution, we are interested in the corresponding lattice of tau functions (4.7).

We make the change of variables $t \mapsto \tau$, where

$$
t=\frac{\theta\left(-1 ; p^{6}\right)^{4}}{\theta\left(-p^{3} ; p^{6}\right)^{4}}, \quad p=e^{\pi \mathrm{i} \tau} .
$$

In terms of the function (2.6),

$$
t=\frac{\zeta(\zeta+2)^{3}}{(2 \zeta+1)^{3}}
$$

Note that the cusps (2.16) correspond precisely to the singular points $t=0,1, \infty$ of (4.1), with one trigonometric and one hyperbolic cusp at each singular point. Substituting (4.13) into (4.12), one finds the modular solution

$$
q=\frac{\zeta(\zeta+2)}{2 \zeta+1}
$$

4.4. Modular tau functions. Although our solution is modular for $\Gamma_{0}(6,2)$, the corresponding tau functions are only modular for a subgroup. The minimal field $\mathcal{M}$ containing all modular functions that we need is generated by

$$
\phi_{1}=\frac{\eta(\tau / 2)^{2}}{\eta(\tau)^{2}}, \quad \phi_{2}=\frac{\eta(2 \tau)^{2}}{\eta(\tau)^{2}}, \quad \phi_{3}=\frac{\eta(3 \tau / 2)}{\eta(\tau / 2)}, \quad \phi_{4}=\frac{\eta(6 \tau)}{\eta(2 \tau)}, \quad \phi_{5}=\frac{\eta(3 \tau)}{\eta(\tau)},
$$

where

$$
\eta(\tau)=e^{\frac{\pi \mathbf{i} \tau}{12}} \prod_{k=1}^{\infty}\left(1-p^{2 k \pi \mathrm{i} \tau}\right)
$$

is Dedekind's eta function. These functions are modular for the group $K$, consisting of transformations (2.15) with

$$
a \equiv d \equiv \pm 1 \bmod 12, \quad b \equiv 0 \bmod 24, \quad c \equiv 0 \bmod 72 .
$$

One can show that $\mathcal{M}$ contains $\mathbb{C}(\zeta)$ and that $\phi_{j}^{12} \in \mathbb{C}(\zeta)$ for each $j$. Thus, with

$$
\delta=t(t-1) \frac{d}{d t}=\frac{\zeta(\zeta+1)(\zeta-1)(\zeta+2)}{2(2 \zeta+1)^{2}} \frac{d}{d \zeta}
$$

we have $\delta\left(\phi_{j}\right) / \phi_{j}=\delta\left(\phi_{j}^{12}\right) / 12 \phi_{j}^{12} \in \mathbb{C}(\zeta)$. In particular, $\mathcal{M}$ is closed under $\delta$. 
The normalizer of $K$ in $\operatorname{SL}(2, \mathbb{Z})$ is $\Gamma_{0}(3)$, which is generated by $T(\tau)=\tau+1$ and $U(\tau)=(\tau-1) /(3 \tau-2)$. Let $t_{1}=\left(U T^{3}\right)^{3}$ and $t_{3}=\left(T^{3} U\right)^{3}$. We use the same notation as for Bäcklund transformations in view of the following result.

Proposition 4.1. The following equations define a field automorphism $\mathcal{F} \rightarrow \mathcal{M}$ :

$$
\begin{array}{rlrl}
\mathbf{X}\left(\alpha_{0}\right)=\mathbf{X}\left(\alpha_{1}\right)=\mathbf{X}\left(\alpha_{3}\right)=\mathbf{X}\left(\alpha_{4}\right)=0, & \mathbf{X}\left(\alpha_{2}\right)=\frac{1}{2}, \\
\mathbf{X}(p)=\frac{2 \zeta+1}{2(1-\zeta)(\zeta+2)}, & \mathbf{X}(q)=\frac{\zeta(\zeta+2)}{2 \zeta+1}, & \mathbf{X}(t)=\frac{\zeta(\zeta+2)^{3}}{(1+2 \zeta)^{3}}, \\
\mathbf{X}(u)=\frac{\phi_{1}^{2} \phi_{3}^{4}}{2^{2 / 3} \phi_{5}^{4}}, & \mathbf{X}(v)=-\frac{2^{4 / 3} \phi_{2}^{2} \phi_{4}^{4}}{\phi_{5}^{4}}, & \\
\mathbf{X}\left(\tau_{0}\right)=\frac{1}{\phi_{5}}, & \mathbf{X}\left(\tau_{1}\right)=-\frac{\phi_{3} \phi_{4}}{\phi_{5}^{2}}, & \mathbf{X}\left(\tau_{2}\right)=\frac{2^{-2 / 3} \phi_{5}^{4}}{\phi_{1}^{2} \phi_{2}^{2} \phi_{3}^{2} \phi_{4}^{2}} \\
\mathbf{X}\left(\tau_{3}\right)=\frac{e^{\frac{\pi \mathrm{i}}{4}} \phi_{5}}{\phi_{3}}, & \mathbf{X}\left(\tau_{4}\right)=\frac{e^{\frac{3 \pi \mathrm{i}}{4}} \phi_{5}}{\phi_{4}} . &
\end{array}
$$

This extension satisfies (4.4), as well as the identities

$$
\begin{gathered}
\mathbf{X} \circ t_{j}=t_{j} \circ \mathbf{X}, \quad j=1,3, \\
\mathbf{X} \circ s_{j}=\mathbf{X}, \quad j=0,1,3,4 .
\end{gathered}
$$

This gives a convenient realization of all tau functions corresponding to (4.12) as modular functions, with the Bäcklund transformations $t_{j}$ acting by modular transformations.

4.5. Identification of tau functions. We are now ready to formulate the main result of 8 , which identifies the functions $t^{(\mathbf{k})}$ with Painlevé tau functions. We need the normalizing factor

$$
\begin{aligned}
\phi_{l_{1} l_{2} l_{3} l_{4}}= & \frac{\left.(-1)^{\left(\begin{array}{c}
l_{1}+1 \\
3
\end{array}\right)+\left(\begin{array}{c}
l_{3}+1 \\
3
\end{array}\right)+\left(\begin{array}{c}
l_{4}+1 \\
3
\end{array}\right)+\left(\left(\begin{array}{c}
l_{3}+1 \\
2
\end{array}\right)+l_{1} l_{3}+l_{2}\right) l_{4}} \mathrm{i}_{2}^{l_{3}+1}\right)+\left(\begin{array}{c}
l_{4}+1 \\
2
\end{array}\right)-l_{1}^{2} l_{3}+l_{1} l_{4}^{2}+l_{2}+l_{3}+l_{4}}{2^{l_{0}\left(l_{0}-1\right)+l_{1}^{2}+l_{3}^{2}+l_{4}^{2}}} \\
& \times \zeta^{l_{4}^{2}-l_{0}\left(l_{0}-1\right)-\left(l_{0}+l_{2}\right)\left(l_{2}+l_{4}\right)}(\zeta+1)^{l_{3}^{2}-l_{0}\left(l_{0}-1\right)-\left(l_{0}+l_{2}\right)\left(l_{2}+l_{3}\right)} \\
& \times(\zeta-1)^{\left(l_{0}+l_{2}\right)\left(l_{1}+l_{4}\right)-\left(l_{2}+l_{3}\right)^{2}-l_{3}}(\zeta+2)^{-3 l_{2}\left(l_{0}+l_{2}+l_{4}\right)-\left(l_{0}+l_{4}\right)\left(l_{4}+1\right)} \\
& \times(2 \zeta+1)^{-l_{0}^{2}-l_{1}\left(l_{0}+l_{1}+3 l_{2}+1\right)-l_{2}} u^{\frac{1}{2}\left(l_{1}-l_{3}\right)\left(l_{1}+l_{3}+2 l_{4}-1\right)+2 l_{2}\left(l_{0}+l_{2}\right)} \\
& \times v^{\frac{1}{2}\left(l_{1}-l_{4}\right)\left(l_{1}+l_{4}+2 l_{3}-1\right)+2 l_{2}\left(l_{0}+l_{2}\right)} \tau_{0}^{l_{0}+1} \tau_{1}^{l_{1}} \tau_{2}^{l_{2}} \tau_{3}^{l_{3}} \tau_{4}^{l_{4}} .
\end{aligned}
$$

Recall also the sequence $Y_{k}$ defined in (2.17).

Theorem 4.2. We have

$$
\mathbf{X}\left(\frac{\tau_{l_{1} l_{2} l_{3} l_{4}}}{\phi_{l_{1} l_{2} l_{3} l_{4}}}\right)=Y_{k_{0}} Y_{k_{1}} Y_{k_{2}} Y_{k_{3}} t^{\left(k_{0}, k_{1}, k_{2}, k_{3}\right)}
$$


where

$$
k_{0}=-l_{1}-l_{2}-l_{4}, \quad k_{1}=-l_{2}, \quad k_{2}=-l_{1}-l_{2}-l_{3}, \quad k_{3}=-l_{2}-l_{3}-l_{4} .
$$

Note that (4.16) defines a bijection between $\mathbb{Z}^{4}$ and the sublattice defined by $\sum k_{j} \in 2 \mathbb{Z}$. To prove Theorem 4.2 we check that, if one would define $t^{(\mathbf{k})}$ using (4.15), all the properties of Theorem 3.3 would hold. This is in principle straightforward.

Let

$$
q_{l_{1} l_{2} l_{3} l_{4}}=\mathbf{X}\left(T_{1}^{l_{1}} T_{2}^{l_{2}} T_{3}^{l_{3}} T_{4}^{l_{4}} q\right) \in \mathbb{C}(\zeta) .
$$

Then $q=q_{l_{1} l_{2} l_{3} l_{4}}$ solves (4.1), with $t$ given by (4.13) and

$$
(\alpha, \beta, \gamma, \delta)=\left(\frac{l_{1}^{2}}{2},-\frac{l_{4}^{2}}{2}, \frac{l_{3}^{2}}{2}, \frac{1-l_{0}^{2}}{2}\right) .
$$

By (4.6), this solution can be factored in terms of the functions $t^{(\mathbf{k})}$. To give an example,

$$
q_{-1,-2,3,-1}=\frac{\zeta(\zeta+2)\left(\zeta^{3}+3 \zeta^{2}+3 \zeta+5\right)\left(5 \zeta^{3}+15 \zeta^{2}+7 \zeta+1\right)}{(2 \zeta+1)\left(5 \zeta^{3}+3 \zeta^{2}+3 \zeta+1\right)\left(\zeta^{3}+7 \zeta^{2}+15 \zeta+5\right)},
$$

where the non-trivial factors can be recognized from

$$
\begin{aligned}
t^{(4,1,-1,0)} & =-\frac{4 \zeta^{10}(\zeta-1)}{(\zeta+1)(\zeta+2)^{10}(2 \zeta+1)}\left(\zeta^{3}+3 \zeta^{2}+3 \zeta+5\right), \\
t^{(3,2,0,-1)} & =\frac{4(\zeta-1)(\zeta+2)^{4}(2 \zeta+1)}{\zeta^{4}(\zeta+1)}\left(5 \zeta^{3}+15 \zeta^{2}+7 \zeta+1\right), \\
t^{(4,1,0,-1)} & =\frac{4 \zeta^{4}(\zeta-1)}{(\zeta+1)(\zeta+2)^{3}(2 \zeta+1)^{8}}\left(5 \zeta^{3}+3 \zeta^{2}+3 \zeta+1\right), \\
t^{(3,2,-1,0)} & =-\frac{4 \zeta^{12}(\zeta-1)}{(\zeta+1)(\zeta+2)^{11}(2 \zeta+1)^{4}}\left(\zeta^{3}+7 \zeta^{2}+15 \zeta+5\right) .
\end{aligned}
$$

4.6. Applications. As an application of Theorem 4.2. we can use the results of $\$ 2.7$ to study the behaviour of the solutions (4.17) at the cusps.

Corollary 4.3. Define $\chi(k)$ as 1 for $k$ odd and 0 for $k$ even. Then,

$$
\begin{aligned}
q_{l_{1} l_{2} l_{3} l_{4}} & =\frac{\zeta^{1+\left|l_{0}\right| \delta_{l_{4}, 0}}(\zeta+2)^{1+\chi\left(l_{1}+l_{3}\right)}}{(2 \zeta+1)^{1+\chi\left(l_{3}+l_{4}\right)}} f(\zeta) \\
& =1+\frac{(\zeta+1)^{1+\left|l_{0}\right| \delta_{l_{3}, 0}}(\zeta-1)^{1+\chi\left(l_{1}+l_{4}\right)}}{(2 \zeta+1)^{1+\chi\left(l_{3}+l_{4}\right)}} g(\zeta),
\end{aligned}
$$

with $f$ and $g$ rational functions without zeroes or poles in $\{0,1,-1,-2,-1 / 2\}$. Moreover,

$$
\lim _{\zeta \rightarrow \infty} \zeta^{1+\left|l_{0}\right| \delta_{l_{1}, 0}} q_{l_{1} l_{2} l_{3} l_{4}}
$$

exists and is non-zero. 
Corollary 4.3 encodes the behaviour of the solutions near the singular points of (4.1). For instance, near $t=0,(4.13)$ behaves either as $t \sim \zeta$ or as $t \sim(\zeta+2)^{3}$. The first branch corresponds to $q \sim t^{1+\left|l_{0}\right| \delta_{l_{4}, 0}}$ and the second branch to $q \sim t^{1 / 3}$ or $q \sim t^{2 / 3}$, depending on the parity of $l_{1}+l_{3}$.

We can also apply Theorem 4.2 to deduce new properties of the functions $t^{(\mathbf{k})}$. For instance, when acting on $\mathcal{F}_{0}$, the transformations $\left\{s_{0}, s_{1}, s_{3}, s_{4}, t_{1}, t_{3}\right\}$ generate the group $\mathrm{S}_{4} \times \mathrm{S}_{2} \times \mathrm{S}_{2}$. The property (4.14) can be used to obtain a symmetry of the functions $t^{(\mathbf{k})}$ under that group, which agrees with the total set of symmetries discussed in 92.6 . In particular, this proves Proposition 2.4.

There are many bilinear relations for tau functions (see e.g. [M]) that can be translated to relations for the functions $t^{(\mathbf{k})}$. To give an example,

$$
\begin{aligned}
\frac{\delta^{2}\left(\tau_{l_{1} l_{2} l_{3} l_{4}}\right) \tau_{l_{1} l_{2} l_{3} l_{4}}}{t}-\frac{\delta\left(\tau_{l_{1} l_{2} l_{3} l_{4}}\right)^{2}}{t}- & \delta\left(\tau_{l_{1} l_{2} l_{3} l_{4}}\right) \tau_{l_{1} l_{2} l_{3} l_{4}} \\
& +S\left(l_{0}-\alpha_{0}, l_{1}-\alpha_{1}, l_{3}-\alpha_{3}, l_{4}-\alpha_{4}\right) \tau_{l_{1} l_{2} l_{3} l_{4}}^{2} \\
= & (-1)^{l_{3}+l_{4}} \mathrm{i} u \tau_{l_{1}, l_{2}+1, l_{3}-1, l_{4}} \tau_{l_{1}, l_{2}-1, l_{3}+1, l_{4}}
\end{aligned}
$$

holds as an identity in $\mathcal{F}_{0}$, where

$$
S\left(l_{0}, l_{1}, l_{3}, l_{4}\right)=\frac{1}{12}\left(2 l_{0}^{2}-l_{1}^{2}+2 l_{3}^{2}-l_{4}^{2}+6 l_{0} l_{3}+4 l_{0}+6 l_{3}+2\right) .
$$

This leads to the following result.

Proposition 4.4. The functions $t_{n}=t^{\left(k_{0}+n, k_{1}+n, k_{2}, k_{3}\right)}(\zeta), n \in \mathbb{Z}$, satisfy the recursion

$$
\begin{aligned}
& -\left(2 k_{0}+2 n+1\right)\left(2 k_{1}+2 n+1\right) \frac{(\zeta+2)^{2}}{\zeta^{2}} t_{n+1} t_{n-1} \\
& \quad=A\left(t_{n}^{\prime \prime} t_{n}-\left(t_{n}^{\prime}\right)^{2}\right)+B t_{n}^{\prime} t_{n}+C_{n} t_{n}^{2},
\end{aligned}
$$

where

$$
\begin{aligned}
& A=\zeta(\zeta+1)^{2}(\zeta-1)^{2}(\zeta+2)(2 \zeta+1), \\
& B=2(\zeta+1)^{2}(\zeta-1)\left(\zeta^{3}-3 \zeta^{2}-6 \zeta-1\right),
\end{aligned}
$$

and $C_{n}$ is a polynomial in $k_{j}, n$ and $\zeta$.

We refer to [R5] for the explicit expression for $C_{n}$. Proposition 4.4 settles some conjectures for polynomials related to solvable models. The cases $\mathbf{k}=(0,0,0,0)$ and $(0,0,1,-1)$ prove $[$ BM2, Conj. $1(\mathrm{~b})]$. Moreover, the case $\mathbf{k}=(1,0,0,-1)$ proves [MB, Conj. 6].

Zinn-Justin [Z2] derived recursions for polynomials equivalent to $t^{(0,2 n, 0,0)}$ and $t^{(-1,2 n+1,0,0)}$. These can also be proved using the relation to Painlevé tau functions. In fact, any sequence of the form $t_{n}=t^{\left(k_{0}, k_{1}+2 n, k_{2}, k_{3}\right)}$ satisfies a recursion similar to Proposition 4.4. 
As another application, it follows from (4.10) that $t^{(\mathbf{k})}$ satisfies a quadratic differential equation. This seems to be a novel observation, even in special cases.

Proposition 4.5. The function $t=t^{(\mathbf{k})}(\zeta)$ satisfies a differential equation of the form

$$
\sum_{i \geq j \geq 0, i+j \leq 4} A_{i j} \frac{d^{i} t}{d \zeta^{i}} \frac{d^{j} t}{d \zeta^{j}}=0
$$

with coefficients $A_{i j}$ that are polynomials in $\zeta$ and $k_{0}, \ldots, k_{3}$.

The coefficients are very cumbersome to write down in general. To give a concrete example, consider the case $\mathbf{k}=(0,2 n, 0,0)$. It follows from our results that

$$
t^{(0,2 n, 0,0)}(\zeta)=\left(\frac{\zeta(\zeta+1)}{\zeta+2}\right)^{n(n-1)} f_{n}\left((2 \zeta+1)^{2}\right)
$$

with $f_{n}$ a polynomial of degree $\left(\begin{array}{c}n \\ 2\end{array}\right)$, which is related to the polynomial $q_{n}$ of [MB] by

$$
q_{n}(z)=D_{n} z^{n(n+1)} f_{n+1}\left(z^{-2}\right),
$$

where $D_{n}$ is a constant, see (5.5). In terms of $f_{n}(z)$, (4.21) takes the form

$$
\begin{aligned}
& z(z-1)^{3}(z-9)^{3}\left(f_{n}^{(4)} f_{n}-4 f_{n}^{(3)} f_{n}^{\prime}+3\left(f_{n}^{\prime \prime}\right)^{2}\right) \\
& +(7 z-3)(z-1)^{2}(z-9)^{3}\left(f_{n}^{(3)} f_{n}-f_{n}^{\prime \prime} f_{n}^{\prime}\right) \\
& -2(z-1)(z-9)\left\{(z+1)(z-9)^{2} n^{2}+2(z-9)^{2} n-5 z^{3}+105 z^{2}-483 z+351\right\} f_{n}^{\prime \prime} f_{n} \\
& +2(z-1)(z-9)\left\{(z+1)(z-9)^{2} n^{2}+2(z-9)^{2} n-z^{3}+9 z^{2}-111 z+135\right\}\left(f_{n}^{\prime}\right)^{2} \\
& -\left\{2(z-9)\left(z^{3}-39 z^{2}+139 z+27\right) n^{2}+8(z-9)\left(3 z^{2}+2 z+27\right) n\right. \\
& \left.-2 z^{4}+72 z^{3}-876 z^{2}+2184 z-1890\right\} f_{n}^{\prime} f_{n} \\
& -2 n(n-1)\left\{(5 z-21)(z-9) n^{2}-(z+15)(z-9) n+z^{2}+22 z+9\right\} f_{n}^{2}=0 .
\end{aligned}
$$

As a final remark, we stress that the functions $t_{n}^{\left(k_{0}, k_{1}, k_{2}, k_{3}\right)}$ are defined by explicit determinants. For instance, for $n \geq 0$,

$$
\begin{aligned}
& t^{(n, n, 0,0)}=\lim _{\substack{x_{1}, \ldots, x_{n} \rightarrow \xi_{0} \\
y_{1}, \ldots, y_{n} \rightarrow \xi_{1}}} \frac{\prod_{i, j=1}^{n} G\left(x_{i}, y_{j}\right)}{\prod_{1 \leq i<j \leq n}\left(y_{j}-y_{i}\right)\left(x_{j}-x_{i}\right)} \operatorname{det}_{1 \leq i, j \leq n}\left(\frac{1}{G\left(x_{i}, y_{j}\right)}\right), \\
& =\frac{G\left(\xi_{0}, \xi_{1}\right)^{n^{2}}}{\prod_{j=1}^{n}(j-1) !^{2}} \operatorname{det}_{1 \leq i, j \leq n}\left(\left.\frac{\partial^{i+j-2}}{\partial x^{i-1} \partial y^{j-1}}\right|_{x=\xi_{0}, y=\xi_{1}} \frac{1}{G(x, y)}\right) .
\end{aligned}
$$

We have proved that these functions solve the recursion (4.20). This is reminiscent of how the Toda equation

$$
\tau_{n+1} \tau_{n-1}=\tau_{n}^{\prime \prime} \tau_{n}-\left(\tau_{n}^{\prime}\right)^{2}
$$


is solved by Hankel determinants

$$
\tau_{n}=\operatorname{det}_{1 \leq i, j \leq n}\left(f^{(i+j-2)}\right) .
$$

However, an important difference is that, whereas (4.23) is immediately obtained from (4.24) by applying the Jacobi-Desnanot identity, that is not the case for (4.22). It must be combined with the Schrödinger equation, which allows us to replace the derivatives in $x$ and $y$ with $\zeta$-derivatives (cf. \$3.2).

It should be mentioned that genuine Hankel determinants for tau functions of Painlevé VI have been given in [KM]. These are different in nature from (4.22). It would be interesting to know whether identities such as (4.22) are peculiar to our choice of seed solution, or if similar formulas can be found in other situations.

\section{Comparison OF NOTATiON}

In this Section, we explain how $T_{n}^{(\mathbf{k})}$ are related to various polynomials appearing in [BM1, BM2, $\mathrm{BH}, \mathrm{FH}, \mathrm{H}, \mathrm{MB}, \mathrm{RS}, \mathrm{R} 2, \mathrm{Z2}$.

5.1. Polynomials related to the three-colour model. In [R2], we worked with a symmetric polynomial in $2 n+1$ variables, defined by

$$
\begin{aligned}
& S_{n}\left(x_{1}, \ldots, x_{n}, y_{1}, \ldots, y_{n}, z\right) \\
& =\frac{\prod_{i, j=1}^{n} G\left(x_{i}, y_{j}\right)}{\prod_{1 \leq i<j \leq n}\left(x_{j}-x_{i}\right)\left(y_{j}-y_{i}\right)} \operatorname{det}_{1 \leq i, j \leq n}\left(\frac{F\left(x_{i}, y_{j}, z\right)}{G\left(x_{i}, y_{j}\right)}\right),
\end{aligned}
$$

where $G$ is as in (2.8) and

$$
F(x, y, z)=(\zeta+2) x y z-\zeta(x y+y z+x z+x+y+z)+\zeta(2 \zeta+1) .
$$

It is not hard to prove that

$$
S_{n}\left(x_{1}, \ldots, x_{2 n+1}\right)=\frac{2^{n} \prod_{j=1}^{2 n+1}\left(x_{j}-\zeta\right)}{1-\zeta} T_{n}^{(0,0,0,-1)}\left(x_{1}, \ldots, x_{2 n+1}\right) .
$$

We can then rewrite the polynomials $P_{n}, p_{n}, y_{n}$ and $\tilde{p}_{n}$ of [R2, Prop. 8.1] in terms of $T_{n}^{(\mathbf{k})}$. Namely (recall that $\delta(n)=\left[n^{2} / 4\right]$ ),

$$
\begin{aligned}
P_{n}(x, \zeta) & =\frac{(-1)^{[n / 2]}\left(\frac{\zeta}{2}+1\right)^{n(n-1)-\delta(n-1)}(x-\zeta)}{(1-\zeta) \zeta^{n(n-1)}(\zeta+1)^{n(n-2)}(2 \zeta+1)^{\delta(n-1)}} T_{n}^{(n, n, 0,-1)}(x) \\
p_{n}(\zeta) & =\frac{(-1)^{[n / 2]}\left(\frac{\zeta}{2}+1\right)^{n(n-1)-\delta(n-1)}}{(1-\zeta) \zeta^{n(n-1)}(\zeta+1)^{n^{2}-2 n-1}(2 \zeta+1)^{\delta(n)}} t^{(n+1, n, 0,-1)} \\
y_{n}(\zeta) & =\frac{(-1)^{[n / 2]}\left(\frac{\zeta}{2}+1\right)^{(n-1)^{2}-\delta(n-2)}}{2^{[(n+3) / 2]}(1-\zeta) \zeta^{n(n-1)}(\zeta+1)^{n^{2}-2 n-1}(2 \zeta+1)^{\delta(n+1)}} t^{(n+2, n-1,0,-1)} \\
\tilde{p}_{n}(\zeta) & =\frac{(-1)^{[n / 2]+1} 2^{[(n-1) / 2]}\left(\frac{\zeta}{2}+1\right)^{n^{2}-1-\delta(n)}}{(1-\zeta) \zeta^{n^{2}-1}(\zeta+1)^{n^{2}-2 n-1}(2 \zeta+1)^{\delta(n-1)}} t^{(n, n+1,0,-1)}
\end{aligned}
$$


The main result of [R2] is that the domain wall partition function for the threecolour model can be expressed in terms of $p_{n}$ and $\tilde{p}_{n}$. The polynomial $P_{n}$ is related to the domain wall partition function for the 8VSOS model.

5.2. Polynomials of Bazhanov and Mangazeev. We will now consider the polynomials $\mathcal{P}_{n}(x, z)$ of Bazhanov and Mangazeev [BM1, BM2, MB], which describe the ground state eigenvalue of Baxter's $Q$-operator for the supersymmetric $(\Delta=-1 / 2)$ periodic XYZ chain of odd length. In [BM1], these polynomials are defined up to a factor independent of $x$, and then normalized by writing

$$
\mathcal{P}_{n}(x, z)=\sum_{k=0}^{n} r_{k}^{(n)}(z) x^{k}
$$

and requiring that $r_{n}^{(n)}(0)=1$. Since this only determines $\mathcal{P}_{n}(x, z)$ up to a multiplicative factor $f(z)$ with $f(0)=1$, we make the definition precise by requiring in addition that $\mathcal{P}_{n}(x, z)$ is not divisible by any non-constant polynomial in $z$. We can then prove that $\mathcal{P}_{n}$ is related to (5.1) by

$$
\begin{aligned}
\mathcal{P}_{n}\left(y, \frac{\zeta}{(\zeta+2)(2 \zeta+1)}\right)= & \left(\frac{2}{(\zeta+2)(2 \zeta+1)}\right)^{\delta(n)}\left(\frac{\zeta y+\zeta+2}{\zeta(\zeta+1)}\right)^{n} \\
& \times P_{n}\left(\frac{\zeta(y+2 \zeta+1)}{\zeta y+\zeta+2}, \zeta\right) .
\end{aligned}
$$

This is easy to prove up to a $\zeta$-dependent factor, but to identify that factor we use Theorem 3.2 .

Bazhanov and Mangazeev also introduced the polynomials

$$
\begin{aligned}
& s_{n}(z)=r_{n}^{(n)}(z)=\lim _{x \rightarrow \infty} \frac{\mathcal{P}_{n}(x, z)}{x^{n}}, \\
& \bar{s}_{n}(z)=r_{n}^{(0)}(z)=\mathcal{P}_{n}(0, z),
\end{aligned}
$$

which in our notation is

$$
\begin{gathered}
\left(\frac{(\zeta+2)(2 \zeta+1)}{2}\right)^{\delta(n)} s_{n}\left(\frac{\zeta}{(\zeta+2)(2 \zeta+1)}\right) \\
=\frac{(-1)^{[n / 2]}\left(\frac{\zeta}{2}+1\right)^{n(n-1)-\delta(n-1)}}{\zeta^{n(n-1)}(\zeta+1)^{n(n-1)}(2 \zeta+1)^{\delta(n-1)}} t^{(n, n, 0,0)} \\
\left(\frac{(\zeta+2)(2 \zeta+1)}{2}\right)^{\delta(n)} \bar{s}_{n}\left(\frac{\zeta}{(\zeta+2)(2 \zeta+1)}\right) \\
=\frac{(-1)^{[n / 2]+1} 2^{n-1}\left(\frac{\zeta}{2}+1\right)^{n^{2}-1-\delta(n-1)}}{\zeta^{n^{2}-1}(\zeta+1)^{n(n-1)}(2 \zeta+1)^{\delta(n-1)}} t^{(n, n, 1,-1)}
\end{gathered}
$$


5.3. Polynomials of Zinn-Justin. In [MB], Mangazeev and Bazhanov gave a number of conjectures for eigenvectors of the supersymmetric XYZ Hamiltonian on a periodic chain of odd length. These involve polynomials $p_{n}$ (not to be confused with (5.2)) and $q_{n}$, indexed by $n \in \mathbb{Z}$, which can conjecturally be used to factorize the polynomials $s_{n}$ and $\bar{s}_{n}$. For instance, for $n \geq 0$ it is conjectured that

$$
s_{2 n+1}\left(y^{2}\right)=p_{n}(y) p_{n}(-y) .
$$

Zinn-Justin [Z2] expressed $p_{n}$ and $q_{n}$ in terms of the symmetric polynomials

$$
H_{2 n}\left(x_{1}, \ldots, x_{n}, y_{1}, \ldots, y_{n}\right)=\frac{\prod_{i, j=1}^{n} h\left(x_{i}, y_{j}\right)}{\prod_{1 \leq i<j \leq n}\left(x_{j}-x_{i}\right)\left(y_{j}-y_{i}\right)} \operatorname{det}_{1 \leq i, j \leq n}\left(\frac{1}{h\left(x_{i}, y_{j}\right)}\right),
$$

where

$$
h(x, y)=1-\left(3+\zeta_{\mathrm{Z}}^{2}\right) x y+\left(1-\zeta_{\mathrm{Z}}^{2}\right) x y(x+y)
$$

and $\zeta_{\mathrm{Z}}$ is a parameter (with a subscript to distinguish it from our $\zeta$ ). It is easy to see that

$$
T_{n}\left(\phi\left(x_{1}\right), \ldots, \phi\left(x_{2 n}\right)\right)=\left(\frac{\zeta(\zeta+1)}{\zeta+2}\right)^{n(n-1)} H_{2 n}\left(x_{1}, \ldots, x_{2 n}\right)
$$

where

$$
\phi(x)=\frac{\zeta}{\zeta+2}(1-2(\zeta+1) x)
$$

and $\zeta_{\mathrm{Z}}=2 \zeta+1$

It follows that, in our notation,

$$
p_{n}\left(\frac{1}{2 \zeta+1}\right)=\frac{(-1)^{n} C_{n}(\zeta+2)^{n^{2}-n-1}}{\zeta^{n^{2}-2 n-1}(\zeta+1)^{n(n-1)}(2 \zeta+1)^{n^{2}+n+1}} t^{(-1,2 n+1,0,0)},
$$

where $C_{n}=2^{n}$ for $n \geq 0$ and $C_{n}=3^{n+1} / 2^{n+2}$ for $n \leq-1$. Similarly,

$$
q_{n}\left(\frac{1}{2 \zeta+1}\right)=D_{n}\left(\frac{\zeta+2}{\zeta(\zeta+1)(2 \zeta+1)}\right)^{n(n+1)} t^{(0,2 n+2,0,0)},
$$

where $D_{n}=1$ for $n \geq-1$ and $D_{n}=3^{n+2} / 2^{2 n+3}$ for $n \leq-2$. The identity (5.3), and related conjectures from [MB], can thus be expressed in terms of $t^{(\mathbf{k})}$. We hope to return to these conjectures in the future.

\section{ACKnowledgements}

This research has been supported by the Swedish Science Research Council. I would like to thank Vladimir Bazhanov, Stefan Kolb, Vladimir Mangazeev, Dmitrii Novikov, Bulat Suleimanov and Paul Zinn-Justin for interesting discussions and correspondence. I am also grateful to Nikolai Reshetikhin who suggested that I write the present summary of the work described in [R3, R4, R5]. 


\section{REFERENCES}

[B1] R. J. Baxter, One-dimensional anisotropic Heisenberg chain, Ann. Phys. 70 (1972), 323-337.

[B2] R. J. Baxter, Eight-vertex model in lattice statistics and one-dimensional anisotropic Heisenberg chain II. Equivalence to a generalized ice-type model, Ann. Phys. 76 (1973), 25-47.

[BM1] V. V. Bazhanov and V. V. Mangazeev, Eight-vertex model and non-stationary Lamé equation, J. Phys. A 38 (2005), L145-L153.

[BM2] V. V. Bazhanov and V. V. Mangazeev, The eight-vertex model and Painlevé VI, J. Phys. A 39 (2006), $12235-12243$.

[BH] M. Beccaria and C. Hagendorf, A staggered fermion chain with supersymmetry on open intervals, J. Phys. A 45 (2012), 365201.

[Be] D. Bernard, On the Wess-Zumino-Witten models on Riemann surfaces, Nucl. Phys. B 309 (1988), 145-174.

[C] P. A. Clarkson, Special polynomials associated with rational solutions of the Painlevé equations and applications to soliton equations, Comput. Methods Funct. Theory 6 (2006), 329-401.

[CD] R. Conte and I. Dornic, The master Painlevé VI heat equation, $\backslash$ protect \vrule width0pt \protect \href \{http://arxiv.org/abs/1409.1166\}\{arXiv:1409.1166\}.

[D] G. Darboux, Sur une équation linéaire, C. R. Acad. Sci. Paris 94 (1882), 1645-1648.

[EK] P. I. Etingof and A. A. Kirillov, Representations of affine Lie algebras, parabolic differential equations, and Lamé functions, Duke Math. J. 74 (1994), 585-614.

[ER] B. Eynard and S. Ribault, Lax matrix solution of $c=1$ conformal field theory, J. High Energy Phys. 2014 (2014): 59.

[FLNO] V. A. Fateev, A. V. Litvinov, A. Neveu and E. Onofri, A differential equation for a four-point correlation function in Liouville field theory and elliptic four-point conformal blocks, J. Phys. A 42 (2009), 304011.

[FH] P. Fendley and C. Hagendorf, Ground-state properties of a supersymmetric fermion chain, J. Stat. Mech. 2011 (2011), P02014.

[FS] P. Fendley and H. Saleur, $N=2$ supersymmetry, Painlevé III and exact scaling functions in 2D polymers, Nuclear Phys. B 388 (1992), 609-626.

[F] G. Filali, Elliptic dynamical reflection algebra and partition function of SOS model with reflecting end, J. Geom. Phys. 61 (2011), 1789-1796.

[GIL] O. Gamayun, N. Iorgov and O. Lisovyy, Conformal field theory of Painlevé VI, J. High Energy Phys. 2012 (2012): 038.

[H] C. Hagendorf, Spin chains with dynamical lattice supersymmetry, J. Stat. Phys. 150 (2013), 609-657.

[HF] C. Hagendorf and P. Fendley, The eight-vertex model and lattice supersymmetry, J. Stat. Phys. 146 (2012), $1122-1155$.

[ICK] A. G. Izergin, D. A. Coker, V. E. Korepin, Determinant formula for the six-vertex model, J. Phys. A 25 (1992) 4315-4334.

[JM] M. Jimbo and T. Miwa, Monodromy preserving deformation of linear ordinary differential equations with rational coefficients. II, Phys. D 2 (1981), 407-448.

[K] V. Kac, Infinite-dimensional Lie Algebras, $3^{\text {rd }}$ edition, Cambridge University Press, 1990.

[KM] K. Kajiwara, T. Masuda, M. Noumi, Y. Ohta and Y. Yamada, Determinant formulas for the Toda and discrete Toda equations, Funkcial. Ekvac. 44 (2001), 291-307.

[Ko] S. Kolb, Radial part calculation for $\widehat{\mathfrak{s l}}_{2}$ and the Heun KZB-heat equation, $\backslash$ protect \vrule width0pt \protect \href\{http://arxiv.org/abs/1310.0782\}\{arXiv:1310.0782\}.

[Ku] G. Kuperberg, Another proof of the alternating-sign matrix conjecture, Internat. Math. Res. Notices 1996 (1996), 139-150.

[LT] E. Langmann and K. Takemura, Source identity and kernel functions for Inozemtsev-type systems, J. Math. Phys. 53 (2012), 082105.

[MB] V. V. Mangazeev and V. V. Bazhanov, Eight-vertex model and Painlevé VI equation. II. Eigenvector results, J. Phys. A 43 (2010), 085206.

[M] T. Masuda, On a class of algebraic solutions to the Painlevé VI equation, its determinant formula and coalescence cascade, Funkcial. Ekvac. 46 (2003), 121-171.

[Ma] M. Mazzocco, Picard and Chazy solutions to the Painlevé VI equation, Math. Ann. 321 (2001), 157-195.

[N1] H. Nagoya, A quantization of the sixth Painlevé equation, in Noncommutativity and Singularities, pp. 291298, Math. Soc. Japan, Tokyo, 2009.

[N2] H. Nagoya, Hypergeometric solutions to Schrödinger equations for the quantum Painlevé equations, J. Math. Phys. 52 (2011), 083509.

[NY] M. Noumi and Y. Yamada, A new Lax pair for the sixth Painlevé equation associated with $\widehat{\mathfrak{s o}}(8)$, in Microlocal Analysis and Complex Fourier Analysis, 238-252, World Sci. Publ., 2002.

[No] D. P. Novikov, The Schlesinger system with $2 \times 2$ matrices and the Belavin-Polyakov-Zamolodchikov equation, Theor. Math. Phys. 161 (2009), 1485-1496. 
[O] S. Okada, Enumeration of symmetry classes of alternating sign matrices and characters of classical groups, J. Algebraic Combin. 23 (2006), 43-69.

[Ok] K. Okamoto, Studies on the Painlevé equations. I. Sixth Painlevé equation $P_{\mathrm{VI}}$, Ann. Mat. Pura Appl. 146 (1987), 337-381.

[P] E. Picard, Mémoire sur la théorie des fonctions algébriques de deux variables, J. Math. Pures Appl. (4) V (1889), 135-319.

[RS] A. V. Razumov and Yu. G. Stroganov, A possible combinatorial point for the XYZ spin chain, Theor. Math. Phys. 164 (2010), 977-991.

[R1] H. Rosengren, An Izergin-Korepin-type identity for the 8VSOS model, with applications to alternating sign matrices, Adv. Appl. Math. 43 (2009), 137-155.

[R2] H. Rosengren, The three-colour model with domain wall boundary conditions, Adv. Appl. Math. 46 (2011), 481-535.

[R3] H. Rosengren, Symmetric polynomials related to the supersymmetric eight-vertex model. I. Behaviour at cusps, \protect \vrule width0pt\protect \href\{http://arxiv.org/abs/1305.0666\}\{arXiv:1305.0666\}.

[R4] H. Rosengren, Symmetric polynomials related to the supersymmetric eight-vertex model. II. Schrödinger equation, \protect \vrule widthOpt\protect \href\{http://arxiv.org/abs/1312.5879\}\{arXiv:1312.5879\}.

[R5] H. Rosengren, Symmetric polynomials related to the supersymmetric eight-vertex model. III. Painlevé VI equation, \protect \vrule width0pt\protect \href\{http://arxiv.org/abs/1405.5318\}\{arXiv:1405.5318\}.

[RS] H. Rosengren and M. Schlosser, Elliptic determinant evaluations and the Macdonald identities for affine root systems, Compositio Math. 142 (2006), 937-961.

[St] Yu. G. Stroganov, The Izergin-Korepin determinant at a cube root of unity, Theor. Math. Phys. 146 (2006), 53-62.

[S1] B. I. Suleimanov, The Hamilton property of Painlevé equations and the method of isomonodromic deformations, Differ. Equ. 30 (1994), 726-732.

[S2] B. I. Suleimanov, "Quantum" linearization of Painlevé equations as a component of their L, A pairs, Ufa Math. J. 4 (2012), 127-136.

[T] O. Tsuchiya, Determinant formula for the six-vertex model with reflecting end, J. Math. Phys. 39 (1998), 5946-5951.

[V] A. P. Veselov, On Darboux-Treibich-Verdier potentials, Lett. Math. Phys. 96 (2011), 209-216.

[ZZ] A. Zabrodin and A. Zotov, Quantum Painlevé-Calogero correspondence for Painlevé VI, J. Math. Phys. 53 (2012), 073508.

[Z1] P. Zinn-Justin, Six-Vertex, Loop and Tiling Models: Integrability and Combinatorics, Habilitation Thesis, Paris, 2008

[Z2] P. Zinn-Justin, Sum rule for the eight-vertex model on its combinatorial line, in Symmetries, Integrable Systems and Representations, 599-637, Springer-Verlag, 2013.

[ZS] A. V. Zotov and A. V. Smirnov, Modifications of bundles, elliptic integrable systems, and related problems, Theor. and Math. Phys. 177 (2013), 1281-1338.

Department of Mathematical Sciences, Chalmers University of Technology and University of

Gothenburg, SE-412 96 Göteborg, Sweden

E-mail address: hjalmar@chalmers.se

$U R L:$ http: //www. math.chalmers.se/ ${ }^{\mathrm{hj} j a l m a r}$ 